The Astrophysical Journal, 600:946-959, 2004 January 10

(C) 2004. The American Astronomical Society. All rights reserved. Printed in U.S.A.

\title{
THE DISTANCES TO OPEN CLUSTERS AS DERIVED FROM MAIN-SEQUENCE FITTING. II. CONSTRUCTION OF EMPIRICALLY CALIBRATED ISOCHRONES ${ }^{1}$
}

\author{
Marc H. Pinsonneault and Donald M. Terndrup \\ Department of Astronomy, The Ohio State University, Columbus, OH 43210; pinsono@astronomy.ohio-state.edu, terndrup@astronomy.ohio-state.edu \\ Robert B. HANSON \\ University of California Observatories/Lick Observatory, Santa Cruz, CA 95064; hanson@ucolick.org \\ AND \\ John R. STAufFer \\ Infrared Processing and Analysis Center, California Institute of Technology, Mail Code 100-22, 770 South Wilson Avenue, Pasadena, CA 91125; \\ stauffer@ipac.caltech.edu \\ Received 2003 August 20; accepted 2003 September 23
}

\begin{abstract}
We continue our series of papers on open cluster distances by comparing multicolor photometry of single stars in the Hyades with theoretical isochrones constructed with various color-temperature relations. After verifying that the isochrone effective temperatures agree well with spectroscopically determined values, we argue that mismatches between the photometry and the theoretical colors likely arise from systematic errors in the colortemperature relations. We then describe a method for empirically correcting the isochrones to match the photometry and discuss the dependence of the isochrone luminosity on metallicity.
\end{abstract}

Subject headings: open clusters and associations: individual (Hyades) — stars: abundances — stars: distances — stars: evolution

\section{INTRODUCTION}

Open clusters are important laboratories for testing stellar evolution models and for deciphering the star formation history of the Galaxy, since each cluster contains samples of stars of a single age and (probably) composition. As is well known, determining distances is the most fundamental step to measuring cluster ages and other key properties.

The success of the Hipparcos mission (ESA 1997) has now provided us with many cluster distances derived from stellar parallaxes. While the new astrometry is a significant advance, it did not come without some controversy. In particular, there were a few cases, notably the Pleiades, in which the Hipparcos parallax distance (Mermilliod et al. 1997; Robichon et al. 1999; van Leeuwen 1999) was significantly different from that obtained by main-sequence (MS) fitting using stellar isochrones (Pinsonneault et al. 1998; Soderblom et al. 1998). A number of papers have discussed these discrepancies in cluster distance estimates, which could have several causes. First, the systematic or random errors in MS-fitting distances could be significantly larger than estimated, as claimed by Robichon et al. (2000), or the photometry, reddening, and/or metal abundances in the best-studied clusters could be seriously in error. In the case of the Pleiades, the cluster may have an unusually high helium abundance, making its main sequence $\approx 0.3$ mag fainter than would be expected from its metallicity (Belikov et al. 1998); this, however, is difficult to understand since there do not seem to be nearby field stars of similar

\footnotetext{
${ }^{1}$ This publication makes use of data products from the Two Micron All Sky Survey, which is a joint project of the University of Massachusetts and the Infrared Processing and Analysis Center/California Institute of Technology, funded by the National Aeronautics and Space Administration and the National Science Foundation.
}

characteristics in the Hipparcos catalog (Soderblom et al. 1998). Alternatively, the metal abundance from spectroscopy may have been overestimated (Percival, Salaris, \& Kilkenny 2003 and references therein); this point has been disputed by, inter alia, Stello \& Nissen (2001). Second, there could be some overlooked flaw in the stellar models used to compute isochrones or in the transformation of model quantities to broadband colors. Particularly on the lower MS in young clusters, stellar activity producing spots or chromospheric emission can affect colors in the blue (van Leeuwen, Alphenaar, \& Meys 1987; Stauffer et al. 2003). Finally, the cluster parallax discrepancies, amounting to $\sim 1$ mas, may just reflect the actual size of Hipparcos' parallax errors over the small angular scale $\left(\lesssim 1^{\circ}\right)$ subtended by most open clusters except the Hyades. On scales comparable with Hipparcos' 0.9 field of view, the individual stellar parallaxes are correlated (van Leeuwen \& Evans 1998), causing local zero-point errors in the cluster parallaxes. These have been suggested to be $\sim 1$ mas in the Pleiades (Makarov 2002) and Coma Ber (Makarov 2003), but smaller in the Hyades (Narayanan \& Gould 1999a; Narayanan \& Gould 1999b; de Bruijne, Hoogerwerf, \& de Zeeuw 2001). Recently, there has been a similar discussion about the distance to NGC 2516 (Terndrup et al. 2002; Percival, Salaris, \& Kilkenny 2003); here uncertainties in the cluster metallicity and in the amount of foreground extinction complicate the comparison between the MS-fitting distance and the Hipparcos measurement. Regardless of the source or sources of these discrepancies, it remains the case that the 1997-1999 analysis of Hipparcos parallaxes and current stellar evolutionary models are not mutually consistent when applied to the ensemble of nearby clusters with precise parallaxes.

The idea that MS-fitting can be used to find distances to clusters and even individual stars has, of course, been around for a long time (e.g., Eggen 1948; Johnson \& Knuckles 1955), 
but it has proven difficult to develop a precise technique using theoretical isochrones, even for determining relative distances $^{2}$ (VandenBerg et al. 2000). The stellar evolution models themselves are now well constrained in the case of the Sun (e.g., Basu, Pinsonneault, \& Bahcall 2000; Bahcall, Pinsonneault, \& Basu 2001), but they are less certain for the full range of temperatures and luminosities in any open cluster. In order to generate isochrones, the models need transformations from theoretical quantities $\left(L, T_{\text {eff }}\right)$ into observational magnitudes and colors. A number of different color-temperature calibrations are available, including some generated empirically (e.g., Gratton, Carretta, \& Castelli 1996; Weiss \& Salaris 1999; Lejeune \& Schaerer 2001; VandenBerg $\&$ Clem 2003). Despite improvements in the determination of atmospheric opacities, bolometric corrections, and so on, there typically remain small but significant mismatches between calculated and isochrone colors and the best photometry in open clusters (e.g., Terndrup et al. 2000; de Bruijne et al. 2001; Castellani, Degl'Innocenti, \& Prada Moroni 2001; Grocholski \& Sarajedini 2003). The size of these discrepancies can be as large as $0.04-0.06$ mag in broadband colors. Furthermore, the dependence on metallicity of the absolute magnitude $M_{V}$ of the MS is a function of color, being greater when bluer colors such as $B-V$ are used in the color-magnitude diagram (CMD) and less, for example, when $V-I$ or $V-K$ are employed. Taken together, all this means that the derived distance depends on the selection of colors used in the MSfitting (Pinsonneault et al. 1998). On the other hand, if the isochrones can be accurately calibrated over a wide range of colors, it may be possible to use multiband photometry to derive more accurate distance and reddening estimates and to provide a photometric abundance indicator for open clusters.

Motivated by the continuing discussion of open cluster distances, we have undertaken a careful reexamination of all the ingredients of the MS-fitting technique, revising and extending our previous formulation (Pinsonneault et al. 1998) and taking advantage of new calibrations from Hipparcos and from improvements to our stellar evolution models. Our first paper (Pinsonneault et al. 2003, hereafter Paper I) discussed the construction of the isochrones and demonstrated that they provided a good match to the mass/luminosity relation for the components of the eclipsing binary vB 22 in the Hyades. In this paper, we perform additional external checks on the metallicity and temperature scales adopted for the Hyades, then use photometry to generate empirical adjustments to the isochrone colors and bolometric corrections. We conclude by discussing the sensitivity of the MS luminosity to metallicity for various available color calibrations. In subsequent papers of this series, we will extend the calibration to fainter magnitudes, provide a grid of calibrated isochrones appropriate for open clusters, and determine the effects of random and systematic errors in the MS fitting method.

\section{THE EMPIRICAL CALIBRATION}

\subsection{Data}

We adopted the Hyades as the zero point for the MS-fitting method for a number of reasons. The cluster has a negligible reddening $E(B-V)=0.003 \pm 0.002$ (Crawford 1975; Taylor $1980)$ and excellent membership information from proper

\footnotetext{
${ }^{2}$ Alternative methods using field stars with measured parallaxes have equivalent challenges; see, for example, VandenBerg et al. (2000).
}

motions and radial velocities. Although the cluster's size is a significant fraction of its distance, the Hipparcos parallaxes and proper motions allow the determination of the distances to individual Hyades members to a precision of $\sim 2 \%$ (de Bruijne et al. 2001), with reasonable assumptions about the cluster's internal motions. The average distance, taken as that of the cluster's center of mass $(m-M=3.33 \pm 0.01$, or $d=46.34 \pm 0.27 \mathrm{pc}$ ) from Perryman et al. (1998), is not altered significantly by correlations of the Hipparcos parallaxes on small angular scales (Narayanan \& Gould 1999b; de Bruijne et al. 2001). The most significant problem is that the Hipparcos data do not extend below about $M_{V}=8$, insufficient for calibrating isochrones for the lower MS. This will be corrected by making use of data in other clusters such as the Pleiades or Praesepe, as discussed in a future paper.

We chose as our Hyades sample a set of stars identified as members of the cluster by Perryman et al. (1998), excluding any star that is a known or suspected binary. Most of these are included in the high-resolution spectroscopic sample of Paulson, Sneden, \& Cochran (2003, hereafter PSC).

To construct a calibrating set of photometry for the Hyades, we inspected the numerous $U B V$ photometric studies and selected those which were in mutual agreement. For $B$ and $V$, we selected stars from Johnson \& Knuckles (1955), Mendoza (1967), and the Hipparcos mission (ESA 1997). In $V$, we also had photometry from Weis and collaborators (Upgren \& Weis 1977; Weis, Delucca, \& Upgren 1979) that was also used for (Kron) $V-I_{K}$ colors (these will be discussed below). The Hipparcos catalog provides individual errors for stars, and the quoted errors in the Johnson and Mendoza papers, respectively, are 0.004 and $0.01 \mathrm{mag}$. The dispersion in $B-V$ between these three samples is consistent with the quoted errors, and the zeropoint difference for stars in common is negligible (of order $0.001 \mathrm{mag}$ or less).

The Hipparcos catalog lists $V$ magnitudes for all of the stars in our sample; all but 32 of these are derived from groundbased photometry. There is a small $(0.012 \mathrm{mag})$ zero-point offset between the space-based Hipparcos $V$ magnitudes and the Johnson/Mendoza data, with dispersions of 0.013 and $0.009 \mathrm{mag}$, respectively; the difference is in the sense that the ground-based data are fainter. There are only six stars in common between Weis and Johnson, with a formal dispersion of $0.015 \mathrm{mag}$ and a zero-point offset of 0.005 (Johnson fainter), which is not statistically significant. We chose the groundbased data for our zero-point, adding $0.012 \mathrm{mag}$ to the Hipparcos space-based $V$ magnitudes, and averaged the results assuming an error of 0.01 for Hipparcos, Mendoza, and Johnson and 0.015 for Weis.

The apparent $V$ magnitudes were converted into absolute $M_{V}$ magnitudes using the individual distance moduli from de Bruijne et al. (2001). The errors for $M_{V}$ are larger than for $V$ (or $V$ used in the construction of $V-K_{s}$ colors), since the error in the distances are included and these errors are usually larger than those in $V$ alone.

We used two sources for the $K$-band data: Carney (1982), which is on the CTIO-CIT system (Frogel et al. 1978; Elias et al. 1982), and data from the Two Micron All Sky Survey (2MASS) All Sky release. The latter is on the "short- $K$ " $\left(K_{s}\right)$ system (Persson et al. 1998; Carpenter 2001). We converted the Carney (1982) $K$ magnitudes to $K_{S}$ using the transformation in Carpenter (2001) and averaged these with the 2MASS values, using Carney's error estimate of 0.011 mag for each star. The error in the $V-K$ color was obtained by adding the errors in $V$ and $K_{s}$ in quadrature. 
The merger of the $I$-band data for the clusters was somewhat more involved than for $B V K$, largely because of the need to transform different $I$-band systems (Johnson, Kron/Eggen, and Cousins) onto the Cousins system. There were three main sources of Johnson data, with a total of 60 stars: Mendoza (1967), Johnson et al. (1966), and Johnson, MacArthur, \& Mitchell (1968). In addition, there were six stars from Carney \& Aaronson (1979).

Weis and collaborators (Upgren \& Weis 1977; Weis et al. 1979; Weis \& Upgren 1982; Upgren, Weis, \& Hanson 1985; Weis \& Hanson 1988) have $V-I$ data in the Kron system for 23 stars. There were also 11 stars with Johnson $V-I$ and accurate $R-I$ colors from Eggen (1982), which are on the Kron system (Eggen 1975); we found that although the Eggen and Johnson $R-I$ colors could be accurately transformed onto the same system, the $V-I$ colors from Eggen had larger errors. This is probably because Eggen's $V$ magnitudes were not obtained at the same time as $R-I$, so the errors in $V-I$ are much greater than in $R-I$. Only five stars in the sample have $V-I$ colors in both the Kron and Johnson systems. We were unable to achieve a good transformation between the merged colors and those in Taylor \& Joner (1985), so we ignored their measurements.

The Johnson data were transformed onto the Cousins system using the prescription in Bessel (1979), while the Weis and Eggen data were transformed onto the Cousins system using the cubic formula in Bessel \& Weis (1987). The average difference between the transformed Johnson and Weis/Eggen colors are 0.009 and $0.004 \mathrm{mag}$, respectively, with dispersions of 0.012 and $0.013 \mathrm{mag}$. These error estimates are only approximate because the number of stars with multiple $V-I$ measures was small: the bright $(V \lesssim 8)$ stars in the Hyades mostly have Johnson photometry, while the faint stars have Kron photometry and the magnitude range of overlap is limited. We therefore assumed that the colors on both systems were correctly transformed onto the Cousins system and did not apply any further zero-point or slope offsets to the transformed $V-I$ data.

The list of merged photometry is presented as Table 1. The Hipparcos designation for each star is in the first column, while the second lists other names. The remaining columns show the visual magnitude and colors, where the errors represent either quoted errors in the various data sets if only one measurement is available, or the standard deviation if more than one datum was averaged.

For completeness, we have also included the $J-K$ and $H-K$ colors for each star, even though they are not otherwise discussed in this paper (in particular, the metallicity sensitivity of $J-K$ is smaller than for $V-K$, and there is very little variation in the values of $H-K$ for MS stars). Note that several of the brightest stars have large errors in the infrared colors because they are saturated in the 2MASS survey. We plan to discuss the full set of infrared colors and their usefulness for reddening and metallicity determinations in a subsequent paper.

\subsection{Comparing Different Color Calibrations}

In Paper I we described the construction of an isochrone for the Hyades and demonstrated that it compared favorably with the masses and luminosities of the components of the Hyades eclipsing binary vB 22. The parameters for the Hyades depend somewhat on the helium abundance assumed for the Hyades and on the adopted solar model used to calibrate the mixing length parameter for convection. The isochrone used here is the same as in Paper I, namely, constructed from models ignoring microscopic diffusion with $Y=0.273$ and $\alpha=1.72$. The age of the Hyades was taken as $550 \mathrm{Myr}$, appropriate for models lacking convective overshoot (e.g., Perryman et al. 1998). The models used the solar abundance mix of Grevesse \& Noels (1993) and scaled to a Hyades abundance of $[\mathrm{Fe} / \mathrm{H}]=+0.13 \pm$ 0.01 (PSC), using the Sun's relative abundances. The PSC average abundance is quite close to that found by Boesgaard \& Friel (1990); see Perryman et al. (1998) for a summary of previous abundance estimates in the Hyades. The input physics is similar to that used in the recent Yi, Kim, \& Demarque (2003) $Y^{2}$ isochrones for stars of solar mass and above. Our usage of the Saumon, Chabrier, \& Van Horn (1995) equation of state for lower mass MS stars does cause intrinsic differences between our isochrones and theirs for stars below $0.8 \mathrm{M}_{\odot}$.

In Figures 1-3 we compare the theoretical isochrone to the Hyades photometry in Table 1, showing the effect of using several available color-temperature calibrations. Our base case, displayed as the solid line in these figures, uses the corrected color-temperature relation in Lejeune et al. (1998), which employed multicolor data to adjust theoretical flux distributions based on Kurucz and Allard \& Hauschildt (1995) atmospheres. This isochrone matches the photometry reasonably well, although systematic departures from the data are readily apparent especially for the reddest stars. ${ }^{3}$ The three color-magnitude diagrams (CMDs) are in $M_{V}$ and $B-V, V-I_{C}$, and $V-K_{s}$, respectively. The absolute magnitudes were generated from the $V$ magnitudes, and individual kinematic parallaxes from de Bruijne et al. (2001) were adjusted to a common distance modulus of $m-M=3.33 \pm 0.01$ (Perryman et al. 1998).

Also shown in Figures $1-3$ as a short-dashed line is the same isochrone generated with the color-temperature relation in Alonso et al. (1995; 1996); equivalently, the long-dashed line displays the isochrone made with the Lejeune et al. (1998) calibration before their application of empirical corrections to the theoretical colors. Here we will refer to the latter as the uncorrected Lejeune et al. (1998) calibration, although note that their terminology is different.

\subsection{Isochrone Effective Temperatures}

We have just shown that a single theoretical isochrone will produce different loci in the color-magnitude diagram when different color-temperature relations are applied. Before correcting the colors to match the Hyades photometry, it is necessary to do one additional test, namely, to verify that the theoretical quantities generated by the stellar evolution models $\left(L, T_{\text {eff }}, \log g\right)$ are reasonably close to that of the actual cluster, since any errors will be washed away by forcing the isochrone colors to match the photometry. The recent spectroscopic temperature determinations by PSC allow this test to be done with precision.

In both theoretical models and direct parallax measurements, high-metallicity stars appear fainter at a fixed color than lowmetallicity stars. This is partially a stellar interiors effect; increased metal abundance makes stars of fixed mass slightly fainter and significantly cooler, moving them above the MS locus of lower metallicity stars. Increased line blanketing in more metal-rich stars also makes them appear redder at fixed effective temperature. Increasing the helium abundance will

\footnotetext{
3 The various available color-temperature relations differ the most for stars cooler than about $4500 \mathrm{~K}$. The empirically corrected Lejeune et al. (1998) relation, for example, is nearly identical to that presented by Flower (1996), but it is bluer by nearly $0.1 \mathrm{mag}$ at $4000 \mathrm{~K}$.
} 
TABLE 1

Merged Photometry in the Hyades

\begin{tabular}{|c|c|c|c|c|c|c|c|c|c|c|c|c|c|c|c|c|c|}
\hline Hipparcos & Other $\mathrm{ID}^{\mathrm{a}}$ & $V$ & $\sigma(V)$ & $M_{V}$ & $\sigma\left(M_{V}\right)$ & $B-V$ & $\sigma(B-V)$ & $V-I_{C}$ & $\sigma\left(V-I_{C}\right)$ & $K_{s}$ & $\sigma\left(K_{s}\right)$ & $V-K_{s}$ & $\sigma\left(V-K_{s}\right)$ & $J-K_{s}$ & $\sigma\left(J-K_{s}\right)$ & $H-K_{s}$ & $\sigma\left(H-K_{s}\right)$ \\
\hline $13806 \ldots \ldots \ldots \ldots$ & vB 153 & 8.915 & 0.007 & 5.856 & 0.026 & 0.856 & 0.003 & 0.871 & 0.016 & 6.905 & 0.022 & 2.010 & 0.023 & 0.478 & 0.025 & 0.092 & 0.023 \\
\hline $13834 \ldots \ldots \ldots \ldots$ & vB 154 & 5.800 & 0.010 & 3.222 & 0.019 & 0.410 & 0.003 & 0.468 & 0.011 & 4.778 & 0.020 & 1.022 & 0.022 & 0.530 & 0.198 & 0.037 & 0.035 \\
\hline $13976 \ldots \ldots \ldots \ldots$ & G76-49 & 7.970 & 0.010 & 6.131 & 0.019 & 0.926 & 0.015 & $\ldots$ & $\ldots$ & 5.841 & 0.024 & 2.129 & 0.026 & 0.482 & 0.022 & 0.110 & 0.043 \\
\hline $14976 \ldots \ldots \ldots \ldots$ & LTT 11045 & 8.162 & 0.010 & 5.156 & 0.025 & 0.732 & 0.017 & $\ldots$ & $\ldots$ & 6.557 & 0.027 & 1.605 & 0.029 & 0.368 & 0.026 & 0.098 & 0.031 \\
\hline $15563 \ldots \ldots \ldots \ldots$ & G79-28 & 9.650 & 0.010 & 7.187 & 0.028 & 1.130 & 0.015 & $\ldots$ & $\ldots$ & 6.881 & 0.022 & 2.769 & 0.024 & 0.664 & 0.024 & 0.074 & 0.052 \\
\hline $15720 \ldots \ldots \ldots \ldots$ & LP $355-64$ & 11.030 & 0.010 & 8.488 & 0.044 & 1.431 & 0.004 & $\ldots$ & & 7.415 & 0.022 & 3.615 & 0.024 & 0.834 & 0.023 & 0.175 & 0.039 \\
\hline $16529 \ldots \ldots \ldots \ldots$ & vB 4 & 8.890 & 0.010 & 5.765 & 0.032 & 0.845 & 0.002 & 0.856 & 0.016 & 6.907 & 0.016 & 1.983 & 0.019 & 0.467 & 0.014 & 0.111 & 0.010 \\
\hline $17766 \ldots \ldots \ldots \ldots$ & G7-15 & 10.850 & 0.010 & 8.033 & 0.048 & 1.340 & 0.006 & 1.558 & 0.016 & 7.509 & 0.016 & 3.341 & 0.019 & 0.764 & 0.020 & 0.115 & 0.030 \\
\hline $18170 \ldots \ldots \ldots \ldots$ & vB 6 & 5.970 & 0.010 & 2.843 & 0.030 & 0.341 & 0.004 & 0.389 & 0.016 & 5.091 & 0.018 & 0.879 & 0.021 & 0.163 & 0.022 & 0.070 & 0.041 \\
\hline $18322 \ldots \ldots \ldots \ldots$ & L8, G7-34 & 10.120 & 0.010 & 6.779 & 0.053 & 1.070 & 0.008 & 1.124 & 0.020 & 7.574 & 0.021 & 2.546 & 0.023 & 0.607 & 0.020 & 0.118 & 0.024 \\
\hline 18327................. & vB 7 & 8.987 & 0.010 & 5.927 & 0.037 & 0.895 & 0.002 & 0.899 & 0.011 & 6.910 & 0.021 & 2.077 & 0.023 & 0.503 & 0.019 & 0.128 & 0.027 \\
\hline $19082 \ldots \ldots \ldots \ldots$ & L12 & 11.415 & 0.007 & 8.007 & 0.071 & 1.348 & 0.004 & 1.577 & 0.020 & 8.107 & 0.026 & 3.308 & 0.027 & 0.782 & 0.034 & 0.152 & 0.031 \\
\hline $19098 \ldots \ldots \ldots \ldots$ & L10 & 9.310 & 0.015 & 6.032 & 0.044 & 0.893 & 0.005 & 0.896 & 0.016 & 7.254 & 0.022 & 2.056 & 0.027 & 0.494 & 0.030 & 0.111 & 0.039 \\
\hline $19148 \ldots \ldots \ldots \ldots$ & vB 10 & 7.850 & 0.006 & 4.449 & 0.036 & 0.591 & 0.003 & 0.607 & 0.016 & 6.446 & 0.024 & 1.404 & 0.025 & 0.307 & 0.025 & 0.086 & 0.038 \\
\hline 19207.............. & L15 & 10.485 & 0.007 & 7.163 & 0.051 & 1.180 & 0.008 & 1.268 & 0.020 & 7.665 & 0.021 & 2.820 & 0.022 & 0.700 & 0.028 & 0.146 & 0.051 \\
\hline $19263 \ldots \ldots \ldots \ldots$ & $\mathrm{BD}+16^{\circ} 558$ & 9.940 & 0.010 & 6.628 & 0.044 & 1.005 & 0.012 & & & 7.511 & 0.026 & 2.429 & 0.028 & 0.594 & 0.027 & 0.088 & 0.044 \\
\hline $19316 \ldots \ldots \ldots \ldots$ & L14 & 11.277 & 0.006 & 7.871 & 0.074 & 1.328 & 0.003 & 1.558 & 0.016 & 8.046 & 0.027 & 3.231 & 0.028 & 0.772 & 0.033 & 0.125 & 0.045 \\
\hline 19441_............... & $\mathrm{BD}+8^{\circ} 642$ & 10.100 & 0.010 & 7.361 & 0.045 & 1.198 & 0.006 & 1.278 & 0.016 & 7.264 & 0.020 & 2.836 & 0.022 & 0.648 & 0.016 & 0.076 & 0.021 \\
\hline 19781.............. & vB 17 & 8.460 & 0.007 & 4.947 & 0.046 & 0.696 & 0.004 & 0.720 & 0.011 & 6.805 & 0.009 & 1.655 & 0.012 & 0.391 & 0.025 & 0.091 & 0.021 \\
\hline 19786_............ & vB 18 & 8.060 & 0.007 & 4.735 & 0.046 & 0.640 & 0.002 & 0.677 & 0.011 & 6.535 & 0.009 & 1.525 & 0.012 & 0.322 & 0.013 & 0.073 & 0.017 \\
\hline $19789 \ldots \ldots \ldots \ldots$ & vB 16 & 7.057 & 0.006 & 3.283 & 0.037 & 0.420 & 0.003 & 0.451 & 0.016 & 6.005 & 0.021 & 1.052 & 0.022 & 0.202 & 0.017 & 0.077 & 0.023 \\
\hline $19793 \ldots \ldots \ldots \ldots$ & vB 15 & 8.067 & 0.020 & 4.820 & 0.039 & 0.658 & 0.003 & 0.692 & 0.011 & 6.516 & 0.020 & 1.551 & 0.028 & 0.361 & 0.023 & 0.083 & 0.018 \\
\hline $19796 \ldots \ldots \ldots \ldots$ & vB 19 & 7.130 & 0.010 & 3.846 & 0.038 & 0.512 & 0.003 & 0.560 & 0.016 & 5.862 & 0.017 & 1.268 & 0.020 & 0.266 & 0.013 & 0.053 & 0.017 \\
\hline $19808 \ldots \ldots \ldots \ldots$ & vA 68 & 10.725 & 0.050 & 7.424 & 0.085 & 1.205 & 0.005 & 1.288 & 0.011 & 7.680 & 0.009 & 3.045 & 0.050 & 0.757 & 0.025 & 0.140 & 0.013 \\
\hline 19834................ & vA 72 & 11.557 & 0.015 & 8.197 & 0.115 & 1.373 & 0.006 & 1.630 & 0.011 & 8.141 & 0.009 & 3.416 & 0.018 & 0.819 & 0.024 & 0.166 & 0.021 \\
\hline $19877 \ldots \ldots \ldots \ldots \ldots$ & vB 20 & 6.321 & 0.007 & 2.987 & 0.033 & 0.399 & 0.004 & 0.459 & 0.016 & 5.340 & 0.009 & 0.981 & 0.011 & 0.188 & 0.030 & 0.075 & 0.020 \\
\hline 19934_............. & vB 21 & 9.147 & 0.006 & 5.625 & 0.043 & 0.814 & 0.002 & 0.825 & 0.011 & 7.275 & 0.022 & 1.872 & 0.023 & 0.426 & 0.020 & 0.101 & 0.020 \\
\hline $20130 \ldots \ldots \ldots \ldots$ & vB 26 & 8.625 & 0.007 & 5.323 & 0.045 & 0.743 & 0.003 & 0.762 & 0.016 & 6.921 & 0.016 & 1.704 & 0.017 & 0.365 & 0.012 & 0.079 & 0.012 \\
\hline 20146.............. & vB 27 & 8.464 & 0.015 & 5.138 & 0.045 & 0.717 & 0.004 & 0.749 & 0.011 & 6.793 & 0.009 & 1.671 & 0.018 & 0.347 & 0.017 & 0.085 & 0.015 \\
\hline 20219............... & vB 30 & 5.591 & 0.007 & 2.331 & 0.035 & 0.279 & 0.003 & 0.338 & 0.011 & 4.861 & 0.009 & 0.730 & 0.011 & 0.149 & 0.033 & 0.074 & 0.045 \\
\hline $20237 \ldots \ldots \ldots \ldots \ldots$ & vB 31 & 7.467 & 0.006 & 4.200 & 0.034 & 0.566 & 0.004 & 0.622 & 0.016 & 6.124 & 0.018 & 1.343 & 0.019 & 0.271 & 0.013 & 0.060 & 0.024 \\
\hline $20261 \ldots \ldots \ldots \ldots$ & vB 33 & 5.266 & 0.007 & 1.879 & 0.037 & 0.223 & 0.003 & 0.272 & 0.011 & 4.689 & 0.016 & 0.577 & 0.017 & 0.061 & 0.046 & 0.284 & 0.282 \\
\hline $20349 \ldots \ldots \ldots \ldots$ & vB 35 & 6.801 & 0.006 & 3.329 & 0.039 & 0.436 & 0.004 & 0.513 & 0.016 & 5.762 & 0.020 & 1.039 & 0.021 & 0.208 & 0.017 & 0.024 & 0.015 \\
\hline $20350 \ldots \ldots \ldots \ldots$ & vB 36 & 6.807 & 0.006 & 3.457 & 0.035 & 0.441 & 0.004 & 0.498 & 0.016 & 5.746 & 0.016 & 1.061 & 0.017 & 0.198 & 0.015 & 0.048 & 0.010 \\
\hline $20357 \ldots \ldots \ldots \ldots . . . .$. & vB 37 & 6.611 & 0.006 & 3.160 & 0.038 & 0.406 & 0.004 & 0.475 & 0.016 & 5.610 & 0.009 & 1.000 & 0.011 & 0.189 & 0.019 & 0.071 & 0.031 \\
\hline $20480 \ldots \ldots \ldots \ldots$ & vB 42 & 8.854 & 0.010 & 5.338 & 0.048 & 0.758 & 0.002 & 0.786 & 0.016 & 7.121 & 0.016 & 1.733 & 0.019 & 0.410 & 0.011 & 0.076 & 0.011 \\
\hline $20485 \ldots \ldots \ldots \ldots . . . .$. & vB 173 & 10.499 & 0.020 & 7.485 & 0.072 & 1.237 & 0.003 & 1.368 & 0.011 & 7.481 & 0.009 & 3.018 & 0.022 & 0.669 & 0.019 & 0.153 & 0.039 \\
\hline 20491_............. & vB 44 & 7.185 & 0.007 & 3.541 & 0.042 & 0.452 & 0.003 & 0.521 & 0.016 & 6.092 & 0.016 & 1.093 & 0.017 & 0.193 & 0.015 & 0.049 & 0.010 \\
\hline $20492 \ldots \ldots \ldots \ldots$ & vB 46 & 9.117 & 0.006 & 5.731 & 0.054 & 0.865 & 0.003 & 0.871 & 0.011 & 7.153 & 0.009 & 1.964 & 0.011 & 0.466 & 0.011 & 0.110 & 0.016 \\
\hline $20527 \ldots \ldots \ldots \ldots \ldots$ & vA 294 & 10.901 & 0.020 & 7.673 & 0.075 & 1.288 & 0.002 & 1.465 & 0.009 & 7.755 & 0.009 & 3.146 & 0.022 & 0.720 & 0.021 & 0.139 & 0.021 \\
\hline $20542 \ldots \ldots \ldots \ldots$ & vB 47 & 4.800 & 0.010 & 1.526 & 0.038 & 0.156 & 0.003 & 0.179 & 0.016 & 4.400 & 0.010 & 0.400 & 0.014 & 0.147 & 0.268 & 0.099 & 0.210 \\
\hline $20557 \ldots \ldots \ldots \ldots$ & vB 48 & 7.141 & 0.006 & 4.010 & 0.037 & 0.521 & 0.004 & 0.576 & 0.016 & 5.871 & 0.020 & 1.270 & 0.021 & 0.277 & 0.020 & 0.075 & 0.025 \\
\hline $20563 \ldots \ldots \ldots \ldots$ & vB 174 & 9.995 & 0.005 & 6.740 & 0.064 & 1.058 & 0.003 & 1.101 & 0.011 & 7.525 & 0.009 & 2.470 & 0.011 & 0.630 & 0.013 & 0.112 & 0.020 \\
\hline $20567 \ldots \ldots \ldots \ldots$ & vB 51 & 6.971 & 0.006 & 3.461 & 0.047 & 0.443 & 0.004 & 0.517 & 0.016 & 5.880 & 0.009 & 1.090 & 0.011 & 0.167 & 0.014 & 0.043 & 0.016 \\
\hline $20635 \ldots \ldots \ldots \ldots \ldots$ & vB 54 & 4.220 & 0.010 & 0.846 & 0.034 & 0.137 & 0.003 & 0.163 & 0.011 & 4.077 & 0.470 & 0.404 & 0.470 & 0.016 & 0.549 & -0.013 & 0.522 \\
\hline 20641............... & vB 55 & 5.281 & 0.007 & 2.048 & 0.033 & 0.248 & 0.003 & 0.311 & 0.016 & 4.607 & 0.016 & 0.674 & 0.017 & 0.479 & 0.250 & 0.314 & 0.230 \\
\hline $20741 \ldots \ldots \ldots \ldots$ & vB 64 & 8.120 & 0.007 & 4.850 & 0.046 & 0.659 & 0.003 & 0.694 & 0.011 & 6.554 & 0.010 & 1.566 & 0.012 & 0.346 & 0.021 & 0.096 & 0.036 \\
\hline $20762 \ldots \ldots \ldots \ldots$ & vA 407 & 10.475 & 0.007 & 7.118 & 0.062 & 1.146 & 0.002 & 1.230 & 0.016 & 7.743 & 0.010 & 2.732 & 0.013 & 0.637 & 0.046 & 0.122 & 0.037 \\
\hline
\end{tabular}


TABLE 1-Continued

\begin{tabular}{|c|c|c|c|c|c|c|c|c|c|c|c|c|c|c|c|c|c|}
\hline Hipparcos & Other $\mathrm{ID}^{\mathrm{a}}$ & V & $\sigma(V)$ & $M_{V}$ & $\sigma\left(M_{V}\right)$ & $B-V$ & $\sigma(B-V)$ & $V-I_{C}$ & $\sigma\left(V-I_{C}\right)$ & $K_{s}$ & $\sigma\left(K_{s}\right)$ & $V-K_{s}$ & $\sigma\left(V-K_{s}\right)$ & $J-K_{s}$ & $\sigma\left(J-K_{s}\right)$ & $H-K_{s}$ & $\sigma\left(H-K_{s}\right)$ \\
\hline 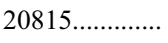 & vB 65 & 7.421 & 0.006 & 4.054 & 0.039 & 0.536 & 0.004 & 0.584 & 0.011 & 6.129 & 0.010 & 1.292 & 0.012 & 0.234 & 0.020 & 0.033 & 0.028 \\
\hline 20826.............. & vB 66 & 7.507 & 0.010 & 4.252 & 0.042 & 0.556 & 0.003 & 0.607 & 0.016 & 6.158 & 0.017 & 1.349 & 0.020 & 0.266 & 0.011 & 0.064 & 0.027 \\
\hline 20827.............. & vB 179 & 9.502 & 0.015 & 6.063 & 0.060 & 0.931 & 0.003 & 0.959 & 0.016 & 7.346 & 0.010 & 2.156 & 0.018 & 0.546 & 0.028 & 0.108 & 0.021 \\
\hline $20842 \ldots \ldots \ldots \ldots \ldots$ & vB 67 & 5.720 & 0.010 & 2.251 & 0.039 & 0.271 & 0.004 & 0.303 & 0.016 & 5.055 & 0.018 & 0.665 & 0.021 & 0.108 & 0.011 & 0.048 & 0.014 \\
\hline $20894 \ldots \ldots \ldots \ldots$ & vB 72 & 3.410 & 0.010 & 0.146 & 0.036 & 0.179 & 0.003 & 0.210 & 0.011 & 2.896 & 0.011 & 0.514 & 0.015 & 0.114 & 0.335 & 0.117 & 0.339 \\
\hline $20899 \ldots \ldots \ldots \ldots . . . . . . .$. & vB 73 & 7.847 & 0.010 & 4.521 & 0.041 & 0.609 & 0.003 & 0.626 & 0.011 & 6.398 & 0.010 & 1.449 & 0.014 & 0.292 & 0.021 & 0.058 & 0.025 \\
\hline 20948................ & vB 78 & 6.914 & 0.010 & 3.609 & 0.042 & 0.451 & 0.002 & 0.516 & 0.009 & 5.791 & 0.010 & 1.123 & 0.014 & 0.236 & 0.029 & 0.078 & 0.034 \\
\hline $20949 \ldots \ldots \ldots \ldots$ & vB 76 & 9.206 & 0.030 & 5.394 & 0.057 & 0.764 & 0.002 & 0.768 & 0.011 & 7.462 & 0.029 & 1.744 & 0.042 & 0.382 & 0.028 & 0.072 & 0.034 \\
\hline $20951 \ldots \ldots \ldots \ldots$ & vB 79 & 8.955 & 0.007 & 5.697 & 0.055 & 0.831 & 0.002 & 0.827 & 0.009 & 7.056 & 0.010 & 1.899 & 0.012 & 0.469 & 0.029 & 0.094 & 0.029 \\
\hline $20978 \ldots \ldots \ldots \ldots . . . . . . . . . .$. & vB 180 & 9.090 & 0.030 & 5.807 & 0.051 & 0.854 & 0.003 & 0.879 & 0.016 & 7.120 & 0.010 & 1.970 & 0.032 & 0.480 & 0.025 & 0.072 & 0.030 \\
\hline $21029 \ldots \ldots \ldots \ldots$ & vB 82 & 4.786 & 0.007 & 1.484 & 0.034 & 0.171 & 0.002 & 0.187 & 0.016 & 4.366 & 0.011 & 0.420 & 0.013 & 0.409 & 0.226 & 0.313 & 0.203 \\
\hline $21036 \ldots \ldots \ldots \ldots$ & vB 84 & 5.406 & 0.007 & 2.159 & 0.036 & 0.262 & 0.003 & 0.311 & 0.016 & 4.732 & 0.009 & 0.674 & 0.012 & 0.473 & 0.312 & 0.086 & 0.034 \\
\hline $21066 \ldots \ldots \ldots \ldots . . . . .$. & vB 86 & 7.045 & 0.007 & 3.746 & 0.040 & 0.465 & 0.004 & 0.529 & 0.016 & 5.893 & 0.020 & 1.152 & 0.021 & 0.246 & 0.032 & 0.051 & 0.028 \\
\hline $21099 \ldots \ldots \ldots \ldots$ & vB 87 & 8.590 & 0.010 & 5.276 & 0.048 & 0.742 & 0.004 & 0.747 & 0.016 & 6.884 & 0.022 & 1.706 & 0.024 & 0.375 & 0.020 & 0.081 & 0.063 \\
\hline $21112 \ldots \ldots \ldots \ldots$ & vB 88 & 7.775 & 0.007 & 4.238 & 0.049 & 0.539 & 0.004 & 0.591 & 0.016 & 6.464 & 0.010 & 1.311 & 0.012 & 0.278 & 0.019 & 0.117 & 0.051 \\
\hline $21138 \ldots \ldots \ldots \ldots$ & vB 191 & 11.055 & 0.015 & 7.678 & 0.121 & 1.293 & 0.003 & 1.471 & 0.016 & 7.895 & 0.024 & 3.160 & 0.028 & 0.772 & 0.024 & 0.139 & 0.020 \\
\hline $21152 \ldots \ldots \ldots \ldots$ & vB 90 & 6.391 & 0.010 & 3.280 & 0.038 & 0.413 & 0.004 & 0.490 & 0.016 & 5.333 & 0.021 & 1.058 & 0.023 & 0.260 & 0.023 & 0.052 & 0.020 \\
\hline $21256 \ldots \ldots \ldots \ldots$ & L66 & 10.687 & 0.015 & 7.515 & 0.060 & 1.236 & 0.004 & 1.362 & 0.016 & 7.686 & 0.026 & 3.001 & 0.030 & 0.741 & 0.026 & 0.149 & 0.025 \\
\hline $21261 \ldots \ldots \ldots \ldots$ & L65 & 10.737 & 0.015 & 7.410 & 0.070 & 1.198 & 0.003 & 1.317 & 0.016 & 7.820 & 0.022 & 2.917 & 0.027 & 0.715 & 0.021 & 0.157 & 0.021 \\
\hline $21267 \ldots \ldots \ldots \ldots . . . . .$. & vB 94 & 6.620 & 0.010 & 3.319 & 0.044 & 0.431 & 0.004 & 0.482 & 0.016 & 5.558 & 0.022 & 1.062 & 0.024 & 0.201 & 0.020 & 0.054 & 0.020 \\
\hline $21317 \ldots \ldots \ldots \ldots$ & vB 97 & 7.920 & 0.010 & 4.633 & 0.046 & 0.635 & 0.004 & 0.638 & 0.016 & 6.454 & 0.010 & 1.466 & 0.014 & 0.300 & 0.022 & 0.107 & 0.034 \\
\hline $21637 \ldots \ldots \ldots \ldots$ & vB 105 & 7.527 & 0.010 & 4.364 & 0.036 & 0.576 & 0.003 & 0.607 & 0.016 & 6.162 & 0.022 & 1.365 & 0.024 & 0.292 & 0.021 & 0.036 & 0.018 \\
\hline $21723 \ldots \ldots \ldots \ldots$ & L80 & 10.043 & 0.015 & 6.847 & 0.066 & 1.072 & 0.004 & 1.136 & 0.016 & 7.480 & 0.022 & 2.563 & 0.027 & 0.609 & 0.021 & 0.122 & 0.024 \\
\hline 21741.............. & vB 109 & 9.397 & 0.007 & 5.495 & 0.058 & 0.812 & 0.002 & 0.816 & 0.011 & 7.565 & 0.020 & 1.832 & 0.021 & 0.431 & 0.017 & 0.066 & 0.015 \\
\hline 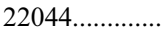 & vB 111 & 5.401 & 0.007 & 2.184 & 0.045 & 0.251 & 0.002 & 0.296 & 0.016 & 4.733 & 0.020 & 0.668 & 0.021 & 0.229 & 0.248 & -0.001 & 0.026 \\
\hline $22177 \ldots \ldots \ldots \ldots$ & L119 & 10.910 & 0.010 & 7.623 & 0.091 & 1.276 & 0.004 & 1.404 & 0.020 & 7.826 & 0.020 & 3.084 & 0.022 & 0.744 & 0.015 & 0.158 & 0.035 \\
\hline $22253 \ldots \ldots \ldots \ldots . . . .$. & L93 & 10.687 & 0.006 & 6.999 & 0.066 & 1.112 & 0.003 & 1.184 & 0.016 & 7.995 & 0.033 & 2.692 & 0.034 & 0.695 & 0.045 & 0.139 & 0.036 \\
\hline $22380 \ldots \ldots \ldots \ldots . . . . .$. & vB 116 & 8.990 & 0.010 & 5.592 & 0.062 & 0.828 & 0.002 & 0.846 & 0.011 & 7.059 & 0.026 & 1.931 & 0.028 & 0.427 & 0.027 & 0.082 & 0.028 \\
\hline $22422 \ldots \ldots \ldots \ldots \ldots$ & vB 118 & 7.737 & 0.010 & 4.327 & 0.048 & 0.580 & 0.003 & 0.607 & 0.016 & 6.355 & 0.016 & 1.382 & 0.019 & 0.287 & 0.015 & 0.078 & 0.017 \\
\hline $22566 \ldots \ldots \ldots \ldots$ & vB 143 & 7.895 & 0.007 & 3.975 & 0.059 & 0.526 & 0.003 & 0.568 & 0.016 & 6.671 & 0.021 & 1.224 & 0.022 & 0.259 & 0.031 & 0.015 & 0.017 \\
\hline $22654 \ldots \ldots \ldots \ldots$ & L98 & 10.290 & 0.010 & 6.704 & 0.081 & 1.070 & 0.008 & 1.114 & 0.020 & 7.742 & 0.020 & 2.548 & 0.022 & 0.633 & 0.025 & 0.143 & 0.026 \\
\hline $22850 \ldots \ldots \ldots \ldots$ & vB 126 & 6.371 & 0.007 & 2.378 & 0.056 & 0.291 & 0.004 & 0.350 & 0.016 & 5.623 & 0.018 & 0.748 & 0.019 & 0.113 & 0.022 & 0.035 & 0.014 \\
\hline $23069 \ldots \ldots \ldots \ldots$ & vB 127 & 8.890 & 0.007 & 5.175 & 0.073 & 0.737 & 0.002 & 0.724 & 0.016 & 7.235 & 0.017 & 1.655 & 0.018 & 0.334 & 0.013 & 0.033 & 0.054 \\
\hline $23214 \ldots \ldots \ldots \ldots$ & vB 128 & 6.755 & 0.007 & 3.593 & 0.039 & 0.450 & 0.004 & 0.498 & 0.016 & 5.645 & 0.016 & 1.110 & 0.017 & 0.211 & 0.018 & 0.049 & 0.030 \\
\hline $23312 \ldots \ldots \ldots \ldots$ & $\mathrm{BD}+04^{\circ} 810$ & 9.722 & 0.010 & 6.159 & 0.074 & 0.957 & 0.047 & 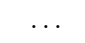 & & 7.589 & 0.026 & 2.133 & 0.028 & 0.524 & 0.026 & 0.103 & 0.039 \\
\hline $23497 \ldots \ldots \ldots \ldots . . . . . . . . . .$. & vB 129 & 4.640 & 0.010 & 1.041 & 0.045 & 0.150 & 0.003 & 0.195 & 0.016 & 4.245 & 0.021 & 0.395 & 0.023 & 0.088 & 0.320 & 0.130 & 0.209 \\
\hline $23750 \ldots \ldots \ldots \ldots$ & $\mathrm{BD}+17^{\circ} 841$ & 8.820 & 0.010 & 5.197 & 0.061 & 0.730 & 0.015 & $\ldots$ & $\ldots$ & 7.128 & 0.017 & 1.692 & 0.020 & 0.386 & 0.011 & 0.088 & 0.044 \\
\hline $24116 \ldots \ldots \ldots \ldots$ & $\mathrm{BD}+20^{\circ} 897$ & 7.852 & 0.010 & 3.314 & 0.084 & 0.445 & 0.015 & $\ldots$ & $\ldots$ & 6.821 & 0.017 & 1.031 & 0.020 & 0.214 & 0.021 & 0.059 & 0.013 \\
\hline $24923 \ldots \ldots \ldots \ldots$ & $\mathrm{BD}+11^{\circ} 772$ & 9.042 & 0.010 & 5.292 & 0.088 & 0.765 & 0.029 & $\ldots$ & $\ldots$ & 7.302 & 0.021 & 1.740 & 0.023 & 0.394 & 0.021 & 0.095 & 0.061 \\
\hline $26382 \ldots \ldots \ldots \ldots$ & vB 168 & 5.541 & 0.006 & 2.014 & 0.072 & 0.222 & 0.004 & 0.257 & 0.016 & 4.935 & 0.025 & 0.606 & 0.026 & 0.127 & 0.027 & 0.067 & 0.031 \\
\hline $28356 \ldots \ldots \ldots \ldots$ & $\mathrm{BD}+02^{\circ} 1102$ & 7.780 & 0.010 & 3.422 & 0.204 & 0.461 & 0.015 & $\ldots$ & $\ldots$ & 6.685 & 0.020 & 1.095 & 0.022 & 0.260 & 0.020 & 0.040 & 0.026 \\
\hline
\end{tabular}

${ }^{\mathrm{a}} \mathrm{G}=$ Giclas, $\mathrm{L}=$ Leiden (Pels), LP = Luyten Palomar, LTT = Luyten Two Tenths, vB = van Buren, and vA = van Altena. 


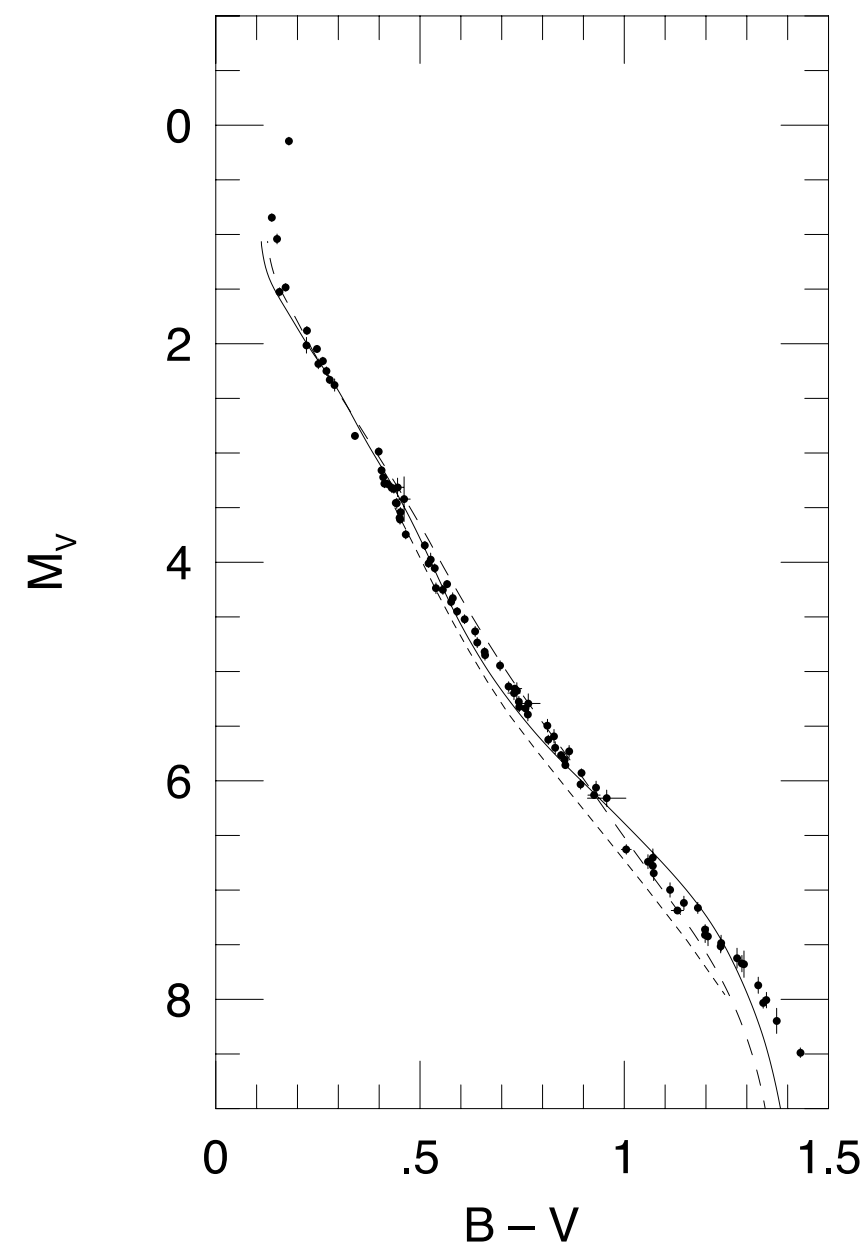

Fig. 1.-Comparison of Hyades single-star photometry ( points) in $M_{V}, B-V$ with a theoretical isochrone (solid line) for $[\mathrm{Fe} / \mathrm{H}]=+0.13$, age $550 \mathrm{Myr}$, which uses the Lejeune et al. (1998) color calibration. Shown as a long-dashed line is the same isochrone, but employing the uncalibrated Lejeune et al. (1998) color-temperature relation; the short-dashed line is the equivalent for the Alonso et al. $(1995,1996)$ calibration. Error bars are shown for all points, even though these are typically smaller than the points themselves.

make stars appear systematically fainter at fixed effective temperature or color; however, the spectral energy distribution and color-temperature relationship is largely insensitive to the helium abundance.

We now proceed to examine the match between the temperature and gravities in the isochrone to parameters derived by PSC. They determined effective temperatures for each star by the requirement that the $\mathrm{Fe}$ I abundances from several lines were independent of excitation potential. Gravity was found from requiring ionization equilibrium that yields the same abundance for $\mathrm{Fe} \mathrm{I}$ and $\mathrm{Fe}$ II lines.

In Figure 4 we display (top) the difference between the PSC temperatures and those from the isochrone with the Lejeune et al. (1998) color calibration, namely, the isochrone plotted as a solid line in Fig. 3. The difference is in the sense of isochrone minus spectra, and the isochrone values for each star were found by looking up the effective temperature at each $M_{V}$. These temperatures are therefore independent of systematic errors in the color calibration, discussed below in $\S 3$. The bottom panel shows the difference in $\log g$ constructed in a similar manner. The circular points show stars that are in our Table 1, while the triangles show other stars in PSC; these were not included in our sample either because they may be binaries or because there was accurate photometry but not accurate luminosities.

Overall, the agreement between the isochrone parameters and the spectroscopically derived values is remarkably good, with average differences of only $-24 \mathrm{~K}$ in $T_{\text {eff }}$ and +0.02 in $\log g$. There are, however, noticeable trends with temperature, in that the PSC temperatures are higher than the isochrone values for the hottest stars in their sample; for these stars, their adopted gravities are somewhat too low. In combination, their temperatures and gravities imply masses about $0.2 M_{\odot}$ above the isochrone values, or radii that are about $5 \%$ too large at fixed mass compared with the isochrones.

In their Table 7, PSC provide coefficients showing the sensitivity of the derived abundances to changes in temperature and gravity, from which we can rescale their $[\mathrm{Fe} / \mathrm{H}]$ to the temperatures and gravities of the isochrones (we adopt their microturbulence values without change). The coefficients were tabulated for two stars at the extremes of the temperature distribution, from which we linearly interpolated to determine the sensitivity of the abundances at any intermediate temperature. Figure 5 (top) displays the PSC values of $[\mathrm{Fe} / \mathrm{H}]$ as a function of isochrone effective temperature, where the symbols are the same as in the previous figure. The rescaled abundances are displayed in the bottom panel.

The mean derived spectroscopic abundance using the rescaled abundances is $\langle[\mathrm{Fe} / \mathrm{H}]\rangle=+0.12 \pm 0.01$. This is in

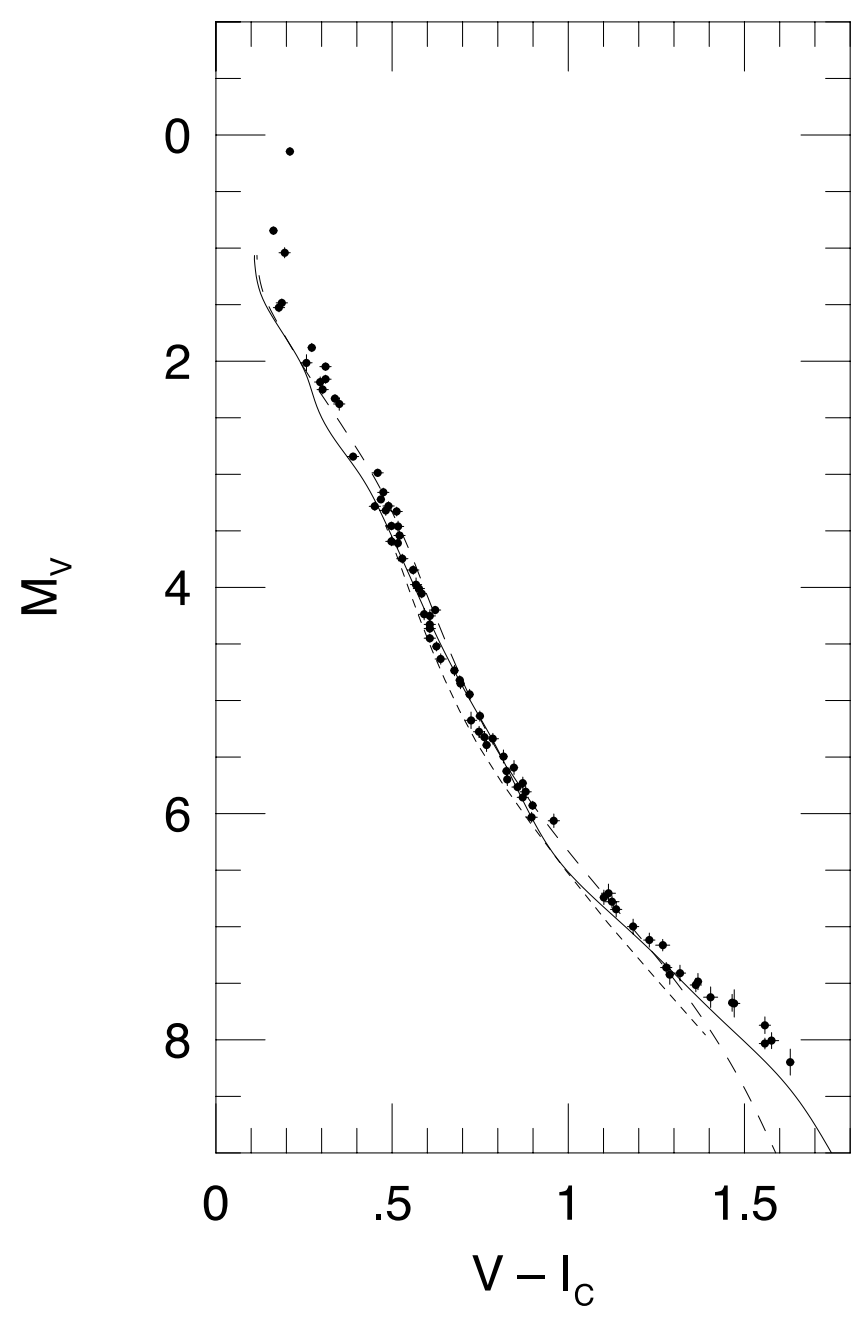

FIG. 2.- Same as Fig. 1, but in $M_{V}, V-I_{C}$ 


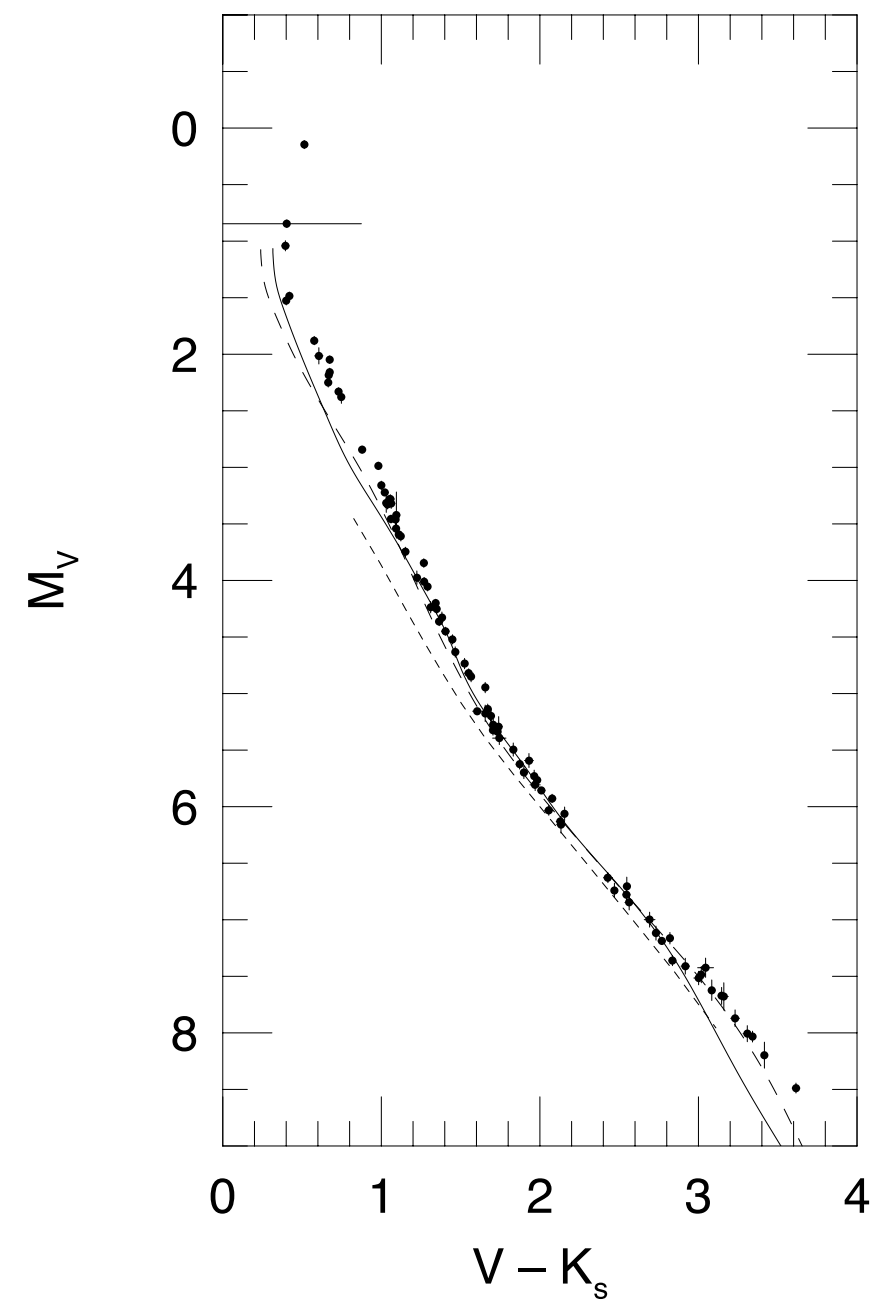

FIG. 3.-Same as Fig. 1, but in $M_{V}, V-K_{s}$

excellent agreement with the original assumed abundance of +0.13 , showing that the locus of the isochrone in the theoretical plane is fully consistent with spectroscopic abundance data to a high degree of accuracy. ${ }^{4}$ The dispersion in the rescaled abundances is slightly smaller than that obtained with the spectroscopic temperatures and gravities, the number of outliers is reduced, and any underlying trends in $[\mathrm{Fe} / \mathrm{H}]$ with $T_{\text {eff }}$ are small. We therefore conclude that the models in the theoretical plane are a reasonable representation of the actual cluster.

\subsection{A Recap}

To summarize, our argument so far has been thus: (1) Solar models are in excellent agreement with the stringent tests possible with helioseismic data (Basu et al. 2000), so models for Hyades stars of similar effective temperatures should be similarly well constrained. (2) The theoretical mass/luminosity relationship is in good agreement with the values obtained from the eclipsing binary $\mathrm{vB} 22$ for a reasonable Hyades helium abundance (Paper I). (3) Available color-temperature relations applied to the same isochrone do not match the photometry of

\footnotetext{
${ }^{4}$ Even though we started with the PSC value in constructing the isochrone, the agreement we find does not amount to a circular argument. If we had adopted (say) $[\mathrm{Fe} / \mathrm{H}]=0.0$ for the isochrone, that isochrone would have been too faint at the Hipparcos distance to the cluster. The temperatures derived from $M_{V}$ would therefore be too high, which would have required the PSC abundances to be increased significantly.
}

the Hyades (§ 2.2). (4) The spectroscopic temperatures of Hyades are consistent with the luminosity/effective temperature relationship in the models $(\S 2.3)$.

We therefore believe that the isochrones in the theoretical luminosity-effective temperature plane are well matched to the Hyades. In other words, the stellar interiors models are not the cause of the mismatch in shape between the isochrones and the Hyades photometry in Figures. 1-3. The deviations for any one isochrone are usually about the same size as the differences between published color calibrations, which suggests that we would be justified in applying modest empirical corrections to the isochrones.

We now proceed to define the empirical locus of the Hyades in the $M_{V}$-color plane and obtain a set of corrections to our base color-temperature relationship (Lejeune et al. 1998). We begin by examining the internal consistency of effective temperatures derived from published color-temperature relationships for the same star but with multiple colors. We then define empirical color-color relationships for the Hyades, tying the other colors to $B-V$ and identifying the locus of the cluster in the $\left(M_{V}, B-V\right)$ plane. When combined with the color-color relationships, this

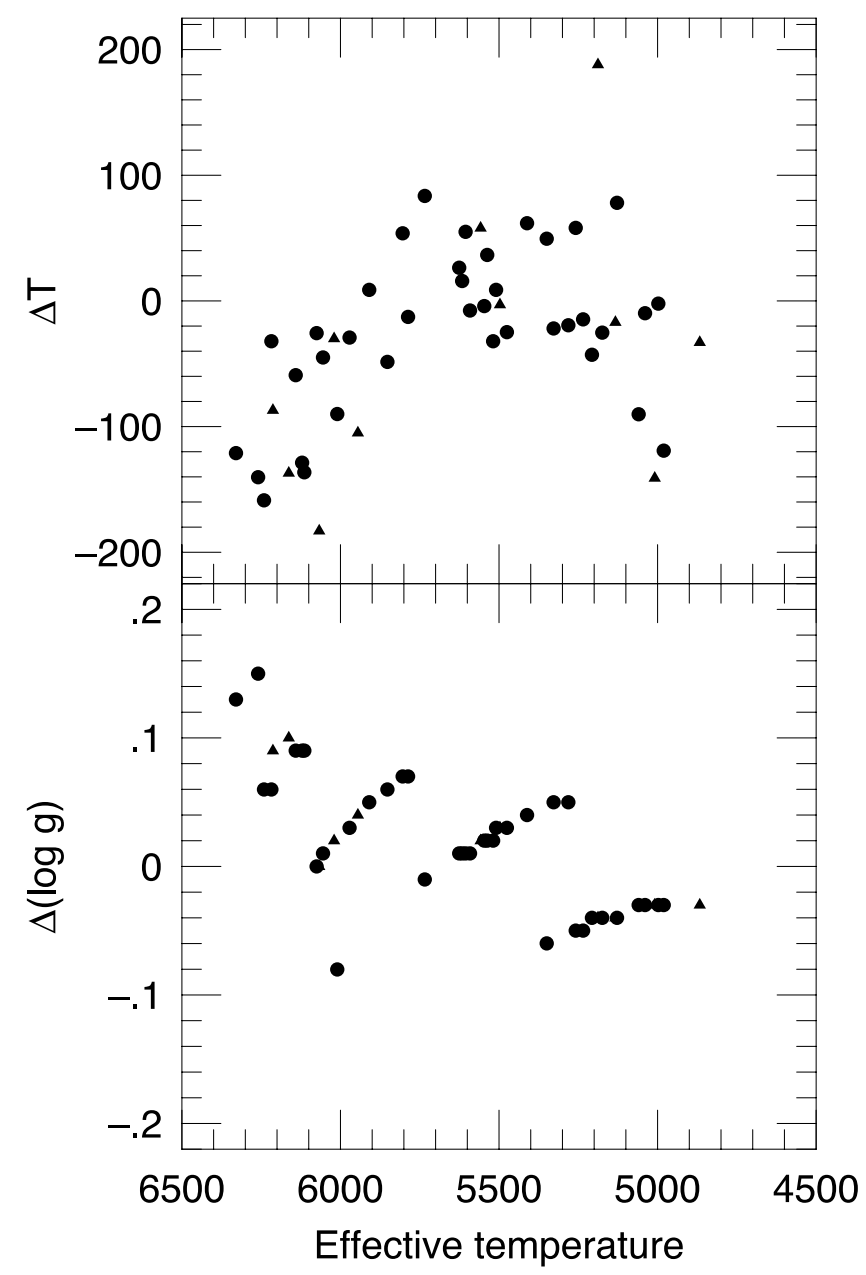

FIG. 4.-Differences in effective temperature (top) and gravity (bottom), in the sense of (ischrone-spectra), between spectroscopically determined parameters (PSC) and the Hyades isochrone at fixed $M_{V}$. While the agreement is good throughout most of the temperature range, the spectroscopic solution yields hotter temperatures and lower gravities than the isochrone. Filled circles are for the stars used to calibrate the empirical color corrections, while the triangles show other stars in the Paulson et al. sample not included in our Table 1. 


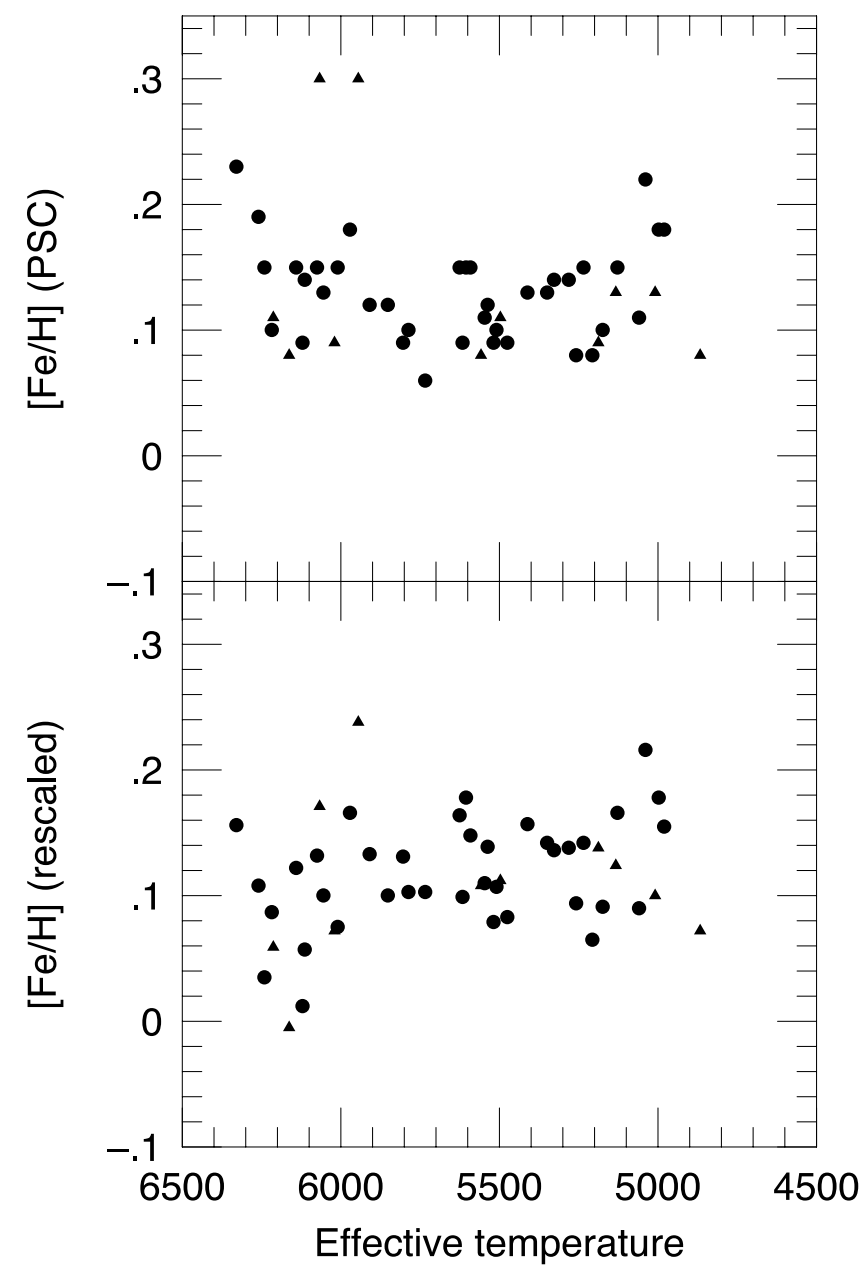

FIG. 5.-Top: Iron abundances for Hyades stars from PSC as a function of isochrone effective temperature. Bottom: Rescaled iron abundances using the isochrone temperatures and gravities, as described in the text. Symbols are the same as in Fig. 4.

defines the locus of the cluster in each of the color indices that we have included in this paper (namely, $B-V, V-I_{C}$, and $V-K_{s}$; other colors will be treated in subsequent papers). Finally, we use the isochrone $M_{V}, T_{\text {eff }}$ relationship to convert these empirical corrections as a function of $M_{V}$ into empirical corrections to the color-temperature relationship.

\subsection{The Internal Consistency of the Color-Temperature Relationships}

If the metallicity and reddening of a cluster are known, a self-consistent set of color-temperature relationships should yield the same temperatures for each star regardless of which color is used to derive the temperature. In Figure 6 we compare the $T_{\text {eff }}$ estimates for each star in Table 1 using the Lejeune et al. (1998) color calibration. The horizontal axis displays the isochrone effective temperature derived from $M_{V}$; note that the temperatures extend to higher values than in Figures 4 and 5 because the PSC survey included only stars cooler than about $T_{\text {eff }}=6300 \mathrm{~K}$. The top panel shows the difference between the temperature estimated from $V-I_{C}$ and that from $B-V$; the middle and bottom panels, respectively, compare the temperature from $V-K_{S}$ to $B-V$ and $V-I_{C}$, again as a function of the temperature from $M_{V}$. Errors in the temperatures are propagated from errors in the photometry.
While the mean temperature differences are nearly zero (the largest is $\langle\Delta T\rangle=-60 \mathrm{~K}$ for the data in the top panel), there are clear systematic differences as a function of temperature that can be as large as $300 \mathrm{~K}$. This indicates that there are temperature ranges where the Lejeune et al. (1998) color calibration is not internally consistent, at least with respect to the Hyades multicolor photometry. These small inconsistencies may have arisen because the color transformation was derived by comparison with an ensemble of field stars with various metallicities; the (generally) small differences noted here would probably not have been apparent in the Lejeune et al. analysis.

We also explored internal inconsistencies using other colortemperature relationships and found similar results. We therefore conclude that the color-temperature calibrations need to be modified not only to remove systematic errors in distances and photometric metallicity, but also for internal consistency. Recently, Grocholski \& Sarajedini (2003) have compared multicolor photometry in many clusters to a number of colortemperature relations and have come to a similar conclusion.

\subsection{Correcting the Color-Color Relationship}

The next step is to correct the color-color relationship. There were several reasons why we performed this correction rather

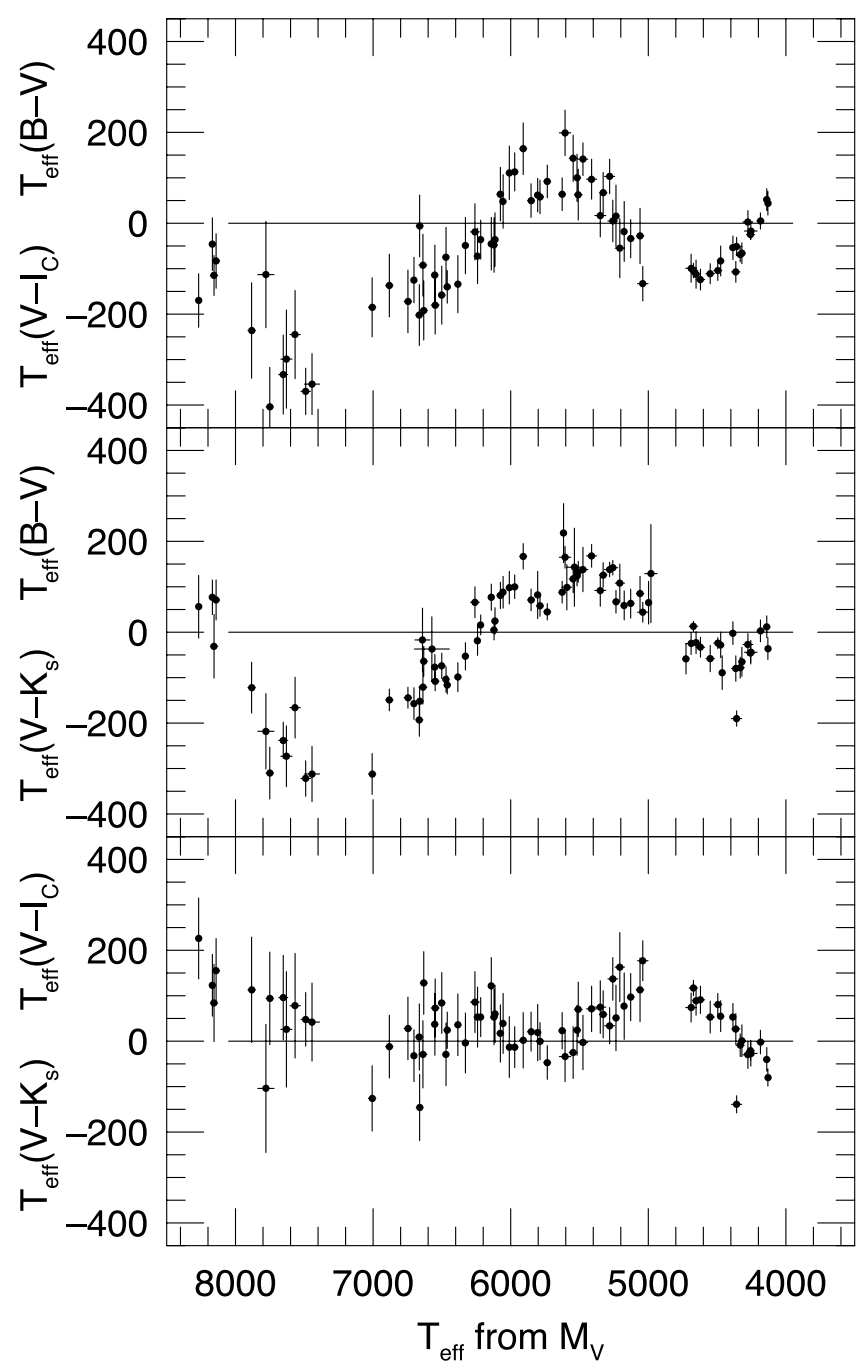

FIg. 6.-Differences between $T_{\text {eff }}$ estimates from $B-V$ and $V-I_{C}$ (top), from $B-V$ and $V-K_{s}$ (middle), and from $V-I_{C}$ and $V-K_{s}$ (bottom) for the Lejeune color calibration as a function of $T_{\text {eff }}$ estimated from the isochrone $M_{V}$. 
than adjusting the isochrones in the $\left(M_{V}\right.$, color) planes individually. First, the errors in the colors are typically smaller than the errors in $M_{V}$; the latter, of course, arise from parallax errors and are much larger than the error in the $V$ magnitudes. Second, correcting the color-color relation necessarily produces the desired result that the isochrone color-temperature relation be internally consistent (i.e., each color yields the same temperature for a star, at least to the limits set by photometric errors).

The smallest observational errors are in $B-V$, so we chose to define corrections in the other colors as a function of $B-V$. The method is illustrated in Figure 7. The top panel shows the color difference $\Delta\left(V-I_{C}\right)$ between the isochrone and the photometry for each star in Table 1, plotted against the observed $B-V$ color. The bottom panel shows the same, but for $V-K_{s}$. The isochrone employed was the same base case (above), namely, for $[\mathrm{Fe} / \mathrm{H}]=+0.13$ and the Lejeune et al. (1998) color calibration; we have neglected systematic errors arising from the small uncertainty in the metallicity of the Hyades. The color differences are in the sense of (photometry - isochrone), so positive values indicate that the star is redder than the isochrone. The isochrone colors $V-I_{C}$ and $V-K_{S}$ were computed by finding the effective temperature at each $B-V$ and then looking up the other colors at that temperature. The error bars on each point include both the observational error in the photometry and the error in the predicted color arising from errors in $B-V$.

The smooth line in each panel of Figure 7 represents the color correction to $V-I_{C}$ or $V-K_{s}$ as a function of $B-V$ (specifically, the $B-V$ color of the Hyades stars). The line was constructed by finding the average value of $\Delta\left(V-I_{C}\right)$ or $\Delta\left(V-K_{s}\right)$ in a moving box containing $3-5$ points sorted by increasing $B-V$. The line was then smoothed by averaging each point with the linear interpolation of adjacent points blueward and redward.

These corrections would generate $V-I_{C}$ and $V-K_{s}$ colors that are consistent with the Hyades photometry once the

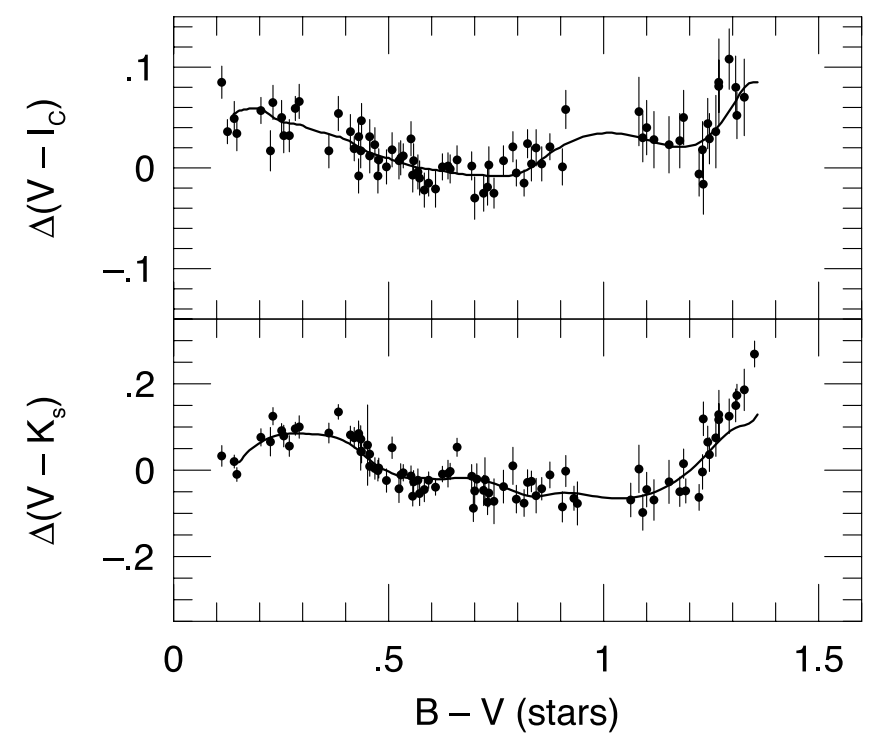

FIG. 7.-Method of constructing color corrections to the theoretical isochrone. The points in the top panel show the difference in $V-I_{C}$ between the isochrone (Fig. 1) and the data at the isochrone color. The sense of the difference is (star-ischrone). The smoothed line was constructed by computing a weighted average difference in a moving window containing $3-5$ stars. The bottom panel is the equivalent for $V-K_{s}$. As discussed in the text, the corrections are applied to the isochrone colors holding $M_{V}$ fixed. isochrone generates the observed $B-V$ colors at the adopted temperature scale. By defining the color corrections in this way, we assume that the $V$-band bolometric corrections in the isochrones are good; $\Delta\left(V-I_{C}\right)$ is then taken as an adjustment to $\mathrm{BC}\left(I_{C}\right)$ and applied to the calculated $I$ magnitude of the isochrone (and equivalently for $B$ and $K_{S}$ ).

The final step is to make the isochrone generate the right $B-V$, as illustrated in Figure 8 , which plots the quantity $\Delta(B-V)$ against the isochrone effective temperature as derived from $M_{V}$. Here the smoothing was done in a moving box of width $200 \mathrm{~K}$ centered at intervals of $100 \mathrm{~K}$, with adjacent pairs averaged.

Applying these corrections to the colors at each effective temperature defines the empirically calibrated isochrone for the Hyades, which is tabulated along with the color corrections in Table 2. The range of colors over which the correction was performed was limited by the following considerations. The Hyades turnoff is around $B-V=0.2$, and the magnitudelimited Hipparcos sample has few stars with $B-V>1.3$. There are only a few stars with $B-V \leq 0.4$, so the correction is not well defined for the bluest colors. Therefore the most reliable color range for the corrections is $0.4 \leq B-V \leq 1.3$.

\section{THE COLOR CALIBRATIONS AND METALLICITY}

The process of empirically correcting the isochrones to match the Hyades necessarily erases many potential systematic errors in the input physics, metallicity scale, or the computation of isochrone colors. On the positive side, the isochrone now matches the shape of the Hyades MS to high precision and (on average) will yield the same effective temperature for the all the various colors. We are thus prepared to derive more accurate distances and metallicities for other open clusters relative to the set of parameters assumed for the Hyades: $[\mathrm{Fe} / \mathrm{H}]=+0.13$, scaled solar abundances, $Y=0.273, \alpha=1.72,\left\langle(m-M)_{0}\right\rangle=$ 3.33 , etc.

There is, however, one more step before we can examine other clusters in detail, namely, measuring the sensitivity of the isochrone luminosity to metallicity in the several colors. For this, we return to the individual color calibrations discussed above. The isochrone absolute magnitude $M_{V}$ depends both on the luminosity of the isochrone in the theoretical plane and on the metallicity sensitivity of the adopted color-temperature relations. We have defined our empirical isochrone with respect to the Lejeune et al. (1998) color-temperature relation and assume that the color corrections displayed in Table 2 are

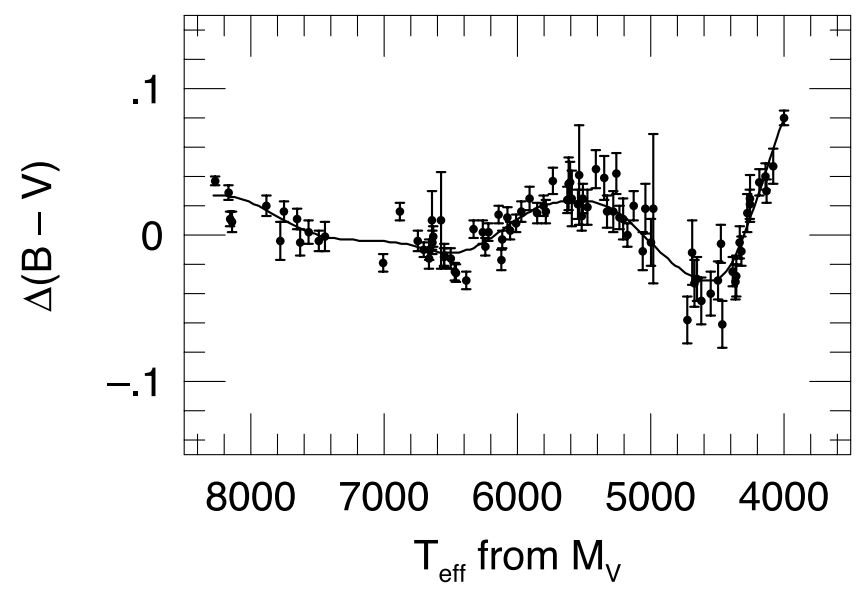

FIG. 8.-Color correction in $B-V$ 
TABLE 2

Empirically Calibrated Hyades Isochrone

\begin{tabular}{|c|c|c|c|c|c|c|c|c|}
\hline $\begin{array}{l}T_{\text {eff }} \\
(\mathrm{K})\end{array}$ & $M / M_{\odot}$ & $M_{V}$ & $B-V$ & $V-I_{C}$ & $V-K_{s}$ & $\Delta(B-V)$ & $\Delta\left(V-I_{C}\right)$ & $\Delta\left(V-K_{s}\right)$ \\
\hline $8280 \ldots \ldots \ldots \ldots$ & 2.235 & 1.10 & 0.139 & 0.162 & 0.374 & 0.027 & 0.053 & 0.014 \\
\hline $8276 \ldots \ldots \ldots \ldots$ & 2.214 & 1.15 & 0.141 & 0.164 & 0.377 & 0.027 & 0.054 & 0.015 \\
\hline $8270 \ldots \ldots \ldots \ldots$ & 2.192 & 1.20 & 0.143 & 0.167 & 0.380 & 0.027 & 0.054 & 0.016 \\
\hline $8262 \ldots \ldots \ldots \ldots$ & 2.170 & 1.25 & 0.146 & 0.170 & 0.384 & 0.027 & 0.055 & 0.017 \\
\hline $8251 \ldots \ldots \ldots \ldots$ & 2.149 & 1.30 & 0.149 & 0.174 & 0.390 & 0.027 & 0.056 & 0.018 \\
\hline $8236 \ldots \ldots \ldots \ldots$ & 2.127 & 1.35 & 0.153 & 0.178 & 0.396 & 0.027 & 0.057 & 0.019 \\
\hline $8215 \ldots \ldots \ldots \ldots$ & 2.105 & 1.40 & 0.158 & 0.184 & 0.409 & 0.027 & 0.057 & 0.024 \\
\hline $8189 \ldots \ldots \ldots \ldots$ & 2.083 & 1.45 & 0.163 & 0.191 & 0.425 & 0.027 & 0.058 & 0.032 \\
\hline $8158 \ldots \ldots \ldots \ldots . . . .$. & 2.062 & 1.50 & 0.170 & 0.199 & 0.443 & 0.026 & 0.058 & 0.040 \\
\hline $8124 \ldots \ldots \ldots \ldots . . . .$. & 2.040 & 1.55 & 0.176 & 0.208 & 0.461 & 0.026 & 0.059 & 0.046 \\
\hline $8088 \ldots \ldots \ldots \ldots$ & 2.019 & 1.60 & 0.183 & 0.218 & 0.479 & 0.025 & 0.059 & 0.051 \\
\hline $8052 \ldots \ldots \ldots \ldots$ & 1.997 & 1.65 & 0.190 & 0.228 & 0.496 & 0.024 & 0.059 & 0.056 \\
\hline $8015 \ldots \ldots \ldots \ldots$ & 1.976 & 1.70 & 0.197 & 0.238 & 0.514 & 0.023 & 0.059 & 0.061 \\
\hline $7978 \ldots \ldots \ldots \ldots$ & 1.955 & 1.75 & 0.204 & 0.247 & 0.532 & 0.021 & 0.058 & 0.065 \\
\hline $7942 \ldots \ldots \ldots \ldots$ & 1.934 & 1.80 & 0.210 & 0.256 & 0.549 & 0.020 & 0.057 & 0.069 \\
\hline $7905 \ldots \ldots \ldots \ldots$ & 1.914 & 1.85 & 0.216 & 0.265 & 0.566 & 0.018 & 0.055 & 0.072 \\
\hline $7868 \ldots \ldots \ldots \ldots$ & 1.893 & 1.90 & 0.223 & 0.273 & 0.582 & 0.016 & 0.053 & 0.075 \\
\hline $7830 \ldots \ldots \ldots \ldots \ldots$ & 1.873 & 1.95 & 0.229 & 0.281 & 0.599 & 0.014 & 0.051 & 0.077 \\
\hline $7790 \ldots \ldots \ldots \ldots . . . .$. & 1.854 & 2.00 & 0.235 & 0.287 & 0.614 & 0.012 & 0.049 & 0.079 \\
\hline $7749 \ldots \ldots \ldots \ldots$ & 1.834 & 2.05 & 0.242 & 0.294 & 0.630 & 0.010 & 0.048 & 0.081 \\
\hline $7706 \ldots \ldots \ldots \ldots . . . .$. & 1.815 & 2.10 & 0.249 & 0.300 & 0.646 & 0.008 & 0.046 & 0.082 \\
\hline $7662 \ldots \ldots \ldots \ldots$ & 1.796 & 2.15 & 0.256 & 0.305 & 0.662 & 0.006 & 0.045 & 0.083 \\
\hline $7616 \ldots \ldots \ldots \ldots$ & 1.776 & 2.20 & 0.263 & 0.310 & 0.678 & 0.004 & 0.045 & 0.084 \\
\hline $7568 \ldots \ldots \ldots \ldots$ & 1.757 & 2.25 & 0.271 & 0.315 & 0.694 & 0.002 & 0.044 & 0.085 \\
\hline $7520 \ldots \ldots \ldots \ldots$ & 1.738 & 2.30 & 0.279 & 0.320 & 0.709 & 0.001 & 0.044 & 0.085 \\
\hline $7471 \ldots \ldots \ldots \ldots$ & 1.720 & 2.35 & 0.286 & 0.324 & 0.724 & -0.001 & 0.043 & 0.085 \\
\hline $7423 \ldots \ldots \ldots \ldots$ & 1.702 & 2.40 & 0.294 & 0.329 & 0.739 & -0.002 & 0.043 & 0.085 \\
\hline $7375 \ldots \ldots \ldots \ldots$ & 1.685 & 2.45 & 0.301 & 0.334 & 0.754 & -0.002 & 0.042 & 0.085 \\
\hline $7327 \ldots \ldots \ldots \ldots$ & 1.667 & 2.50 & 0.308 & 0.341 & 0.769 & -0.003 & 0.040 & 0.084 \\
\hline $7280 \ldots \ldots \ldots \ldots$ & 1.651 & 2.55 & 0.315 & 0.348 & 0.784 & -0.003 & 0.039 & 0.084 \\
\hline $7232 \ldots \ldots \ldots \ldots$ & 1.634 & 2.60 & 0.322 & 0.356 & 0.799 & -0.003 & 0.038 & 0.083 \\
\hline $7186 \ldots \ldots \ldots \ldots . . . .$. & 1.618 & 2.65 & 0.329 & 0.365 & 0.814 & -0.004 & 0.037 & 0.083 \\
\hline $7139 \ldots \ldots \ldots \ldots$ & 1.603 & 2.70 & 0.336 & 0.375 & 0.829 & -0.004 & 0.036 & 0.083 \\
\hline $7092 \ldots \ldots \ldots \ldots$ & 1.587 & 2.75 & 0.343 & 0.386 & 0.845 & -0.004 & 0.035 & 0.082 \\
\hline $7046 \ldots \ldots \ldots \ldots$ & 1.571 & 2.80 & 0.350 & 0.397 & 0.860 & -0.004 & 0.035 & 0.081 \\
\hline $7001 \ldots \ldots \ldots \ldots$ & 1.556 & 2.85 & 0.358 & 0.408 & 0.876 & -0.004 & 0.034 & 0.080 \\
\hline $6956 \ldots \ldots \ldots \ldots . . . . .$. & 1.541 & 2.90 & 0.365 & 0.419 & 0.892 & -0.005 & 0.033 & 0.079 \\
\hline $6913 \ldots \ldots \ldots \ldots$ & 1.526 & 2.95 & 0.372 & 0.429 & 0.909 & -0.005 & 0.032 & 0.077 \\
\hline $6871 \ldots \ldots \ldots \ldots$ & 1.511 & 3.00 & 0.379 & 0.439 & 0.926 & -0.006 & 0.031 & 0.075 \\
\hline $6830 \ldots \ldots \ldots \ldots . . . .$. & 1.496 & 3.05 & 0.387 & 0.449 & 0.943 & -0.006 & 0.031 & 0.072 \\
\hline $6792 \ldots \ldots \ldots \ldots$ & 1.482 & 3.10 & 0.394 & 0.457 & 0.961 & -0.007 & 0.029 & 0.069 \\
\hline $6754 \ldots \ldots \ldots \ldots$ & 1.468 & 3.15 & 0.401 & 0.465 & 0.980 & -0.008 & 0.028 & 0.065 \\
\hline $6718 \ldots \ldots \ldots \ldots . . . .$. & 1.454 & 3.20 & 0.408 & 0.472 & 0.998 & -0.009 & 0.027 & 0.062 \\
\hline $6684 \ldots \ldots \ldots \ldots . . . . .$. & 1.440 & 3.25 & 0.415 & 0.479 & 1.016 & -0.010 & 0.025 & 0.057 \\
\hline $6650 \ldots \ldots \ldots \ldots$ & 1.426 & 3.30 & 0.422 & 0.485 & 1.034 & -0.010 & 0.023 & 0.052 \\
\hline $6618 \ldots \ldots \ldots \ldots \ldots$ & 1.413 & 3.35 & 0.429 & 0.491 & 1.051 & -0.011 & 0.021 & 0.047 \\
\hline $6586 \ldots \ldots \ldots \ldots$ & 1.400 & 3.40 & 0.436 & 0.497 & 1.068 & -0.012 & 0.020 & 0.041 \\
\hline $6556 \ldots \ldots \ldots \ldots$ & 1.387 & 3.45 & 0.443 & 0.503 & 1.084 & -0.012 & 0.018 & 0.034 \\
\hline $6525 \ldots \ldots \ldots \ldots$ & 1.375 & 3.50 & 0.450 & 0.508 & 1.099 & -0.012 & 0.016 & 0.028 \\
\hline $6496 \ldots \ldots \ldots \ldots . . . . .$. & 1.362 & 3.55 & 0.457 & 0.514 & 1.116 & -0.012 & 0.015 & 0.023 \\
\hline $6466 \ldots \ldots \ldots \ldots . . . .$. & 1.350 & 3.60 & 0.464 & 0.521 & 1.132 & -0.012 & 0.014 & 0.017 \\
\hline $6438 \ldots \ldots \ldots \ldots$ & 1.338 & 3.65 & 0.471 & 0.527 & 1.148 & -0.012 & 0.013 & 0.012 \\
\hline $6409 \ldots \ldots \ldots \ldots . . . . .$. & 1.326 & 3.70 & 0.478 & 0.534 & 1.164 & -0.011 & 0.012 & 0.008 \\
\hline $6382 \ldots \ldots \ldots \ldots$ & 1.315 & 3.75 & 0.485 & 0.540 & 1.181 & -0.010 & 0.011 & 0.003 \\
\hline $6354 \ldots \ldots \ldots \ldots$ & 1.303 & 3.80 & 0.493 & 0.546 & 1.197 & -0.010 & 0.010 & 0.000 \\
\hline $6327 \ldots \ldots \ldots \ldots$ & 1.292 & 3.85 & 0.500 & 0.552 & 1.214 & -0.009 & 0.010 & -0.003 \\
\hline $6300 \ldots \ldots \ldots \ldots$ & 1.281 & 3.90 & 0.507 & 0.558 & 1.231 & -0.007 & 0.008 & -0.007 \\
\hline $6273 \ldots \ldots \ldots \ldots$ & 1.269 & 3.95 & 0.515 & 0.564 & 1.247 & -0.006 & 0.007 & -0.010 \\
\hline $6246 \ldots \ldots \ldots \ldots$ & 1.259 & 4.00 & 0.522 & 0.570 & 1.264 & -0.005 & 0.006 & -0.013 \\
\hline $6220 \ldots \ldots \ldots \ldots . . . .$. & 1.248 & 4.05 & 0.530 & 0.576 & 1.281 & -0.003 & 0.005 & -0.015 \\
\hline $6194 \ldots \ldots \ldots \ldots . . . . . . .$. & 1.237 & 4.10 & 0.538 & 0.582 & 1.299 & -0.002 & 0.004 & -0.017 \\
\hline $6167 \ldots \ldots \ldots \ldots . . . . .$. & 1.227 & 4.15 & 0.545 & 0.589 & 1.318 & 0.000 & 0.003 & -0.018 \\
\hline $6141 \ldots \ldots \ldots \ldots . . . .$. & 1.217 & 4.20 & 0.553 & 0.595 & 1.337 & 0.002 & 0.002 & -0.019 \\
\hline $6115 \ldots \ldots \ldots \ldots \ldots$ & 1.207 & 4.25 & 0.561 & 0.601 & 1.357 & 0.003 & 0.001 & -0.018 \\
\hline
\end{tabular}


TABLE 2-Continued

\begin{tabular}{|c|c|c|c|c|c|c|c|c|}
\hline $\begin{array}{l}T_{\text {eff }} \\
(\mathrm{K})\end{array}$ & $M / M_{\odot}$ & $M_{V}$ & $B-V$ & $V-I_{C}$ & $V-K_{s}$ & $\Delta(B-V)$ & $\Delta\left(V-I_{C}\right)$ & $\Delta\left(V-K_{s}\right)$ \\
\hline $6089 \ldots \ldots \ldots \ldots$ & 1.197 & 4.30 & 0.569 & 0.608 & 1.377 & 0.005 & 0.000 & -0.018 \\
\hline $6063 \ldots \ldots \ldots \ldots$ & 1.187 & 4.35 & 0.577 & 0.614 & 1.396 & 0.007 & 0.000 & -0.018 \\
\hline $6036 \ldots \ldots \ldots \ldots$ & 1.177 & 4.40 & 0.585 & 0.621 & 1.414 & 0.009 & -0.001 & -0.018 \\
\hline $6010 \ldots \ldots \ldots \ldots$ & 1.167 & 4.45 & 0.594 & 0.628 & 1.430 & 0.010 & -0.001 & -0.019 \\
\hline $5982 \ldots \ldots \ldots \ldots . .$. & 1.157 & 4.50 & 0.602 & 0.636 & 1.445 & 0.012 & -0.002 & -0.020 \\
\hline $5955 \ldots \ldots \ldots \ldots . . . .$. & 1.147 & 4.55 & 0.611 & 0.643 & 1.460 & 0.013 & -0.002 & -0.021 \\
\hline $5927 \ldots \ldots \ldots \ldots . .$. & 1.137 & 4.60 & 0.619 & 0.651 & 1.474 & 0.015 & -0.003 & -0.021 \\
\hline $5899 \ldots \ldots \ldots \ldots$ & 1.128 & 4.65 & 0.628 & 0.658 & 1.488 & 0.016 & -0.003 & -0.021 \\
\hline 5871............... & 1.118 & 4.70 & 0.637 & 0.666 & 1.503 & 0.018 & -0.004 & -0.020 \\
\hline $5843 \ldots \ldots \ldots \ldots$ & 1.108 & 4.75 & 0.646 & 0.674 & 1.519 & 0.019 & -0.005 & -0.019 \\
\hline $5815 \ldots \ldots \ldots \ldots$ & 1.099 & 4.80 & 0.654 & 0.682 & 1.535 & 0.020 & -0.005 & -0.018 \\
\hline $5787 \ldots \ldots \ldots$ & 1.090 & 4.85 & 0.663 & 0.690 & 1.551 & 0.021 & -0.006 & -0.018 \\
\hline $5760 \ldots \ldots \ldots \ldots$ & 1.080 & 4.90 & 0.672 & 0.698 & 1.568 & 0.021 & -0.006 & -0.018 \\
\hline $5732 \ldots \ldots \ldots \ldots$ & 1.071 & 4.95 & 0.681 & 0.706 & 1.586 & 0.022 & -0.007 & -0.018 \\
\hline $5704 \ldots \ldots \ldots \ldots . .$. & 1.062 & 5.00 & 0.690 & 0.715 & 1.605 & 0.022 & -0.007 & -0.019 \\
\hline $5676 \ldots \ldots \ldots \ldots$ & 1.054 & 5.05 & 0.700 & 0.724 & 1.625 & 0.023 & -0.007 & -0.020 \\
\hline $5648 \ldots \ldots \ldots \ldots$ & 1.045 & 5.10 & 0.709 & 0.733 & 1.646 & 0.023 & -0.007 & -0.021 \\
\hline $5620 \ldots \ldots \ldots \ldots$ & 1.036 & 5.15 & 0.719 & 0.742 & 1.667 & 0.023 & -0.007 & -0.024 \\
\hline $5591 \ldots \ldots \ldots \ldots . .$. & 1.028 & 5.20 & 0.729 & 0.751 & 1.689 & 0.023 & -0.008 & -0.026 \\
\hline $5561 \ldots \ldots \ldots \ldots$ & 1.020 & 5.25 & 0.739 & 0.760 & 1.711 & 0.023 & -0.008 & -0.029 \\
\hline $5532 \ldots \ldots \ldots \ldots$ & 1.012 & 5.30 & 0.749 & 0.768 & 1.733 & 0.023 & -0.008 & -0.032 \\
\hline $5502 \ldots \ldots \ldots \ldots$ & 1.003 & 5.35 & 0.759 & 0.777 & 1.756 & 0.023 & -0.008 & -0.036 \\
\hline $5471 \ldots \ldots \ldots \ldots$ & 0.995 & 5.40 & 0.769 & 0.786 & 1.779 & 0.023 & -0.008 & -0.040 \\
\hline $5440 \ldots \ldots \ldots \ldots$ & 0.988 & 5.45 & 0.780 & 0.795 & 1.802 & 0.023 & -0.008 & -0.044 \\
\hline $5408 \ldots \ldots \ldots \ldots$ & 0.980 & 5.50 & 0.790 & 0.804 & 1.824 & 0.022 & -0.007 & -0.048 \\
\hline 5377................ & 0.972 & 5.55 & 0.801 & 0.813 & 1.847 & 0.021 & -0.006 & -0.052 \\
\hline $5344 \ldots \ldots \ldots \ldots . .$. & 0.964 & 5.60 & 0.811 & 0.823 & 1.870 & 0.020 & -0.004 & -0.056 \\
\hline $5312 \ldots \ldots \ldots \ldots$ & 0.957 & 5.65 & 0.822 & 0.833 & 1.893 & 0.019 & -0.002 & -0.059 \\
\hline $5279 \ldots \ldots \ldots \ldots$ & 0.949 & 5.70 & 0.833 & 0.843 & 1.917 & 0.017 & 0.001 & -0.061 \\
\hline $5245 \ldots \ldots \ldots \ldots$ & 0.942 & 5.75 & 0.844 & 0.854 & 1.941 & 0.015 & 0.004 & -0.061 \\
\hline $5212 \ldots \ldots \ldots \ldots$ & 0.934 & 5.80 & 0.854 & 0.866 & 1.966 & 0.013 & 0.008 & -0.060 \\
\hline $5179 \ldots \ldots \ldots \ldots$ & 0.927 & 5.85 & 0.865 & 0.877 & 1.993 & 0.011 & 0.011 & -0.058 \\
\hline $5146 \ldots \ldots \ldots \ldots$ & 0.920 & 5.90 & 0.876 & 0.888 & 2.019 & 0.008 & 0.015 & -0.056 \\
\hline $5113 \ldots \ldots \ldots \ldots$ & 0.913 & 5.95 & 0.887 & 0.900 & 2.046 & 0.005 & 0.018 & -0.054 \\
\hline $5080 \ldots \ldots \ldots \ldots . . .$. & 0.906 & 6.00 & 0.898 & 0.911 & 2.072 & 0.002 & 0.020 & -0.053 \\
\hline $5048 \ldots \ldots \ldots \ldots$ & 0.899 & 6.05 & 0.908 & 0.921 & 2.098 & -0.001 & 0.023 & -0.052 \\
\hline $5017 \ldots \ldots \ldots \ldots$ & 0.892 & 6.10 & 0.919 & 0.933 & 2.125 & -0.004 & 0.025 & -0.053 \\
\hline $4986 \ldots \ldots \ldots \ldots$ & 0.886 & 6.15 & 0.930 & 0.944 & 2.151 & -0.006 & 0.027 & -0.053 \\
\hline $4956 \ldots \ldots \ldots \ldots . .$. & 0.879 & 6.20 & 0.941 & 0.955 & 2.179 & -0.009 & 0.029 & -0.054 \\
\hline 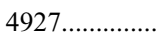 & 0.873 & 6.25 & 0.952 & 0.966 & 2.206 & -0.012 & 0.031 & -0.056 \\
\hline 4898................. & 0.867 & 6.30 & 0.963 & 0.978 & 2.235 & -0.014 & 0.032 & -0.058 \\
\hline $4870 \ldots \ldots \ldots \ldots$ & 0.861 & 6.35 & 0.974 & 0.990 & 2.263 & -0.016 & 0.033 & -0.060 \\
\hline $4843 \ldots \ldots \ldots \ldots$ & 0.855 & 6.40 & 0.985 & 1.003 & 2.293 & -0.019 & 0.034 & -0.061 \\
\hline $4816 \ldots \ldots \ldots \ldots$ & 0.850 & 6.45 & 0.996 & 1.016 & 2.322 & -0.021 & 0.034 & -0.063 \\
\hline $4790 \ldots \ldots \ldots \ldots . . . . .$. & 0.844 & 6.50 & 1.007 & 1.030 & 2.353 & -0.022 & 0.035 & -0.064 \\
\hline $4765 \ldots \ldots \ldots \ldots . .$. & 0.839 & 6.55 & 1.019 & 1.044 & 2.384 & -0.024 & 0.035 & -0.065 \\
\hline $4740 \ldots \ldots \ldots \ldots . . .$. & 0.833 & 6.60 & 1.030 & 1.059 & 2.415 & -0.026 & 0.034 & -0.065 \\
\hline $4715 \ldots \ldots \ldots \ldots$ & 0.828 & 6.65 & 1.041 & 1.074 & 2.446 & -0.027 & 0.034 & -0.065 \\
\hline 4691_................ & 0.823 & 6.70 & 1.053 & 1.090 & 2.478 & -0.028 & 0.033 & -0.065 \\
\hline $4667 \ldots \ldots \ldots \ldots$ & 0.818 & 6.75 & 1.064 & 1.106 & 2.510 & -0.029 & 0.032 & -0.064 \\
\hline 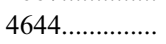 & 0.813 & 6.80 & 1.076 & 1.122 & 2.542 & -0.030 & 0.031 & -0.062 \\
\hline $4620 \ldots \ldots \ldots \ldots$ & 0.808 & 6.85 & 1.087 & 1.138 & 2.574 & -0.031 & 0.030 & -0.060 \\
\hline 4597.................. & 0.803 & 6.90 & 1.099 & 1.155 & 2.606 & -0.031 & 0.028 & -0.057 \\
\hline $4573 \ldots \ldots \ldots$ & 0.798 & 6.95 & 1.110 & 1.171 & 2.638 & -0.031 & 0.027 & -0.054 \\
\hline $4550 \ldots \ldots \ldots \ldots . .$. & 0.793 & 7.00 & 1.121 & 1.187 & 2.670 & -0.031 & 0.025 & -0.050 \\
\hline $4527 \ldots \ldots \ldots \ldots$ & 0.788 & 7.05 & 1.132 & 1.203 & 2.702 & -0.031 & 0.024 & -0.045 \\
\hline $4503 \ldots \ldots \ldots \ldots$ & 0.783 & 7.10 & 1.144 & 1.219 & 2.734 & -0.030 & 0.023 & -0.039 \\
\hline $4480 \ldots \ldots \ldots \ldots$ & 0.778 & 7.15 & 1.155 & 1.235 & 2.765 & -0.029 & 0.021 & -0.033 \\
\hline 4457 ................ & 0.773 & 7.20 & 1.167 & 1.252 & 2.798 & -0.027 & 0.021 & -0.026 \\
\hline $4435 \ldots \ldots \ldots \ldots$ & 0.768 & 7.25 & 1.178 & 1.268 & 2.830 & -0.025 & 0.021 & -0.018 \\
\hline $4412 \ldots \ldots \ldots \ldots$ & 0.763 & 7.30 & 1.190 & 1.285 & 2.862 & -0.022 & 0.021 & -0.009 \\
\hline $4390 \ldots \ldots \ldots \ldots . .$. & 0.757 & 7.35 & 1.202 & 1.302 & 2.894 & -0.019 & 0.022 & 0.000 \\
\hline $4368 \ldots \ldots \ldots \ldots . . . .$. & 0.752 & 7.40 & 1.214 & 1.320 & 2.927 & -0.015 & 0.023 & 0.011 \\
\hline $4347 \ldots \ldots \ldots \ldots$ & 0.747 & 7.45 & 1.226 & 1.339 & 2.961 & -0.010 & 0.026 & 0.023 \\
\hline $4326 \ldots \ldots \ldots \ldots$ & 0.742 & 7.50 & 1.238 & 1.357 & 2.994 & -0.006 & 0.029 & 0.035 \\
\hline $4305 \ldots \ldots \ldots \ldots . . .$. & 0.737 & 7.55 & 1.250 & 1.378 & 3.028 & -0.001 & 0.034 & 0.048 \\
\hline
\end{tabular}


TABLE 2-Continued

\begin{tabular}{|c|c|c|c|c|c|c|c|c|}
\hline $\begin{array}{l}T_{\text {eff }} \\
(\mathrm{K})\end{array}$ & $M / M_{\odot}$ & $M_{V}$ & $B-V$ & $V-I_{C}$ & $V-K_{s}$ & $\Delta(B-V)$ & $\Delta\left(V-I_{C}\right)$ & $\Delta\left(V-K_{s}\right)$ \\
\hline $4285 \ldots \ldots \ldots \ldots$ & 0.731 & 7.60 & 1.262 & 1.400 & 3.061 & 0.004 & 0.039 & 0.061 \\
\hline $4265 \ldots \ldots \ldots \ldots . . .$. & 0.726 & 7.65 & 1.274 & 1.422 & 3.093 & 0.009 & 0.045 & 0.073 \\
\hline $4246 \ldots \ldots \ldots \ldots$ & 0.721 & 7.70 & 1.285 & 1.445 & 3.123 & 0.014 & 0.052 & 0.083 \\
\hline 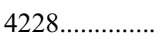 & 0.715 & 7.75 & 1.296 & 1.468 & 3.151 & 0.018 & 0.059 & 0.092 \\
\hline $4209 \ldots \ldots \ldots \ldots$ & 0.710 & 7.80 & 1.307 & 1.493 & 3.177 & 0.023 & 0.066 & 0.098 \\
\hline 4192.............. & 0.705 & 7.85 & 1.317 & 1.517 & 3.199 & 0.028 & 0.074 & 0.102 \\
\hline $4175 \ldots \ldots \ldots \ldots$ & 0.700 & 7.90 & 1.328 & 1.541 & 3.221 & 0.032 & 0.080 & 0.104 \\
\hline $4158 \ldots \ldots \ldots \ldots . .$. & 0.694 & 7.95 & 1.338 & 1.562 & 3.244 & 0.037 & 0.084 & 0.108 \\
\hline $4142 \ldots \ldots \ldots \ldots$ & 0.689 & 8.00 & 1.348 & 1.580 & 3.270 & 0.042 & 0.085 & 0.115 \\
\hline 4126................... & 0.684 & 8.05 & 1.358 & 1.596 & 3.303 & 0.046 & 0.085 & 0.129 \\
\hline $4110 \ldots \ldots \ldots \ldots$ & 0.678 & 8.10 & 1.368 & 1.616 & 3.341 & 0.051 & 0.087 & 0.148 \\
\hline $4095 \ldots \ldots \ldots \ldots$ & 0.673 & 8.15 & 1.377 & 1.643 & 3.385 & 0.055 & 0.098 & 0.172 \\
\hline $4080 \ldots \ldots \ldots \ldots$ & 0.668 & 8.20 & 1.386 & 1.676 & 3.431 & 0.059 & 0.115 & 0.200 \\
\hline $4066 \ldots \ldots \ldots \ldots . . . .$. & 0.663 & 8.25 & 1.395 & 1.711 & 3.477 & 0.063 & 0.135 & 0.226 \\
\hline $4051 \ldots \ldots \ldots \ldots \ldots$ & 0.657 & 8.30 & 1.403 & 1.756 & 3.508 & 0.067 & 0.165 & 0.238 \\
\hline $4038 \ldots \ldots \ldots \ldots . .$. & 0.652 & 8.35 & 1.411 & 1.801 & 3.532 & 0.071 & 0.195 & 0.242 \\
\hline $4024 \ldots \ldots \ldots \ldots$ & 0.647 & 8.40 & 1.419 & 1.844 & 3.554 & 0.074 & 0.224 & 0.244 \\
\hline $4010 \ldots \ldots \ldots \ldots \ldots$ & 0.642 & 8.45 & 1.426 & 1.882 & 3.586 & 0.077 & 0.250 & 0.256 \\
\hline 3997_............... & 0.636 & 8.50 & 1.432 & 1.927 & 3.624 & 0.080 & 0.282 & 0.274 \\
\hline
\end{tabular}

independent of metallicity. As a result, we will be measuring cluster distances on this system and wish to compare this with the available alternatives.

In Figure 9 we illustrate the sensitivity of the MS luminosity to metallicity for the three color-temperature relations we explored in $\S 2.2$. The top panel plots the quantity

$$
\begin{aligned}
& \Delta M_{V}(B-V)=\left\langle M_{V}([\mathrm{Fe} / \mathrm{H}])\right. \\
& \left.-M_{V}([\mathrm{Fe} / \mathrm{H}]=+0.13)\right\rangle\left.\right|_{B-V=0.4-1.0},
\end{aligned}
$$

namely, the average difference in absolute magnitude between the isochrone at any particular metallicity and that for $[\mathrm{Fe} / \mathrm{H}]=+0.13$, computed over the range $0.4 \leq(B-V) \leq$ 1.0 at constant $B-V$. The points are at intervals of 0.1 in metallicity, from $[\mathrm{Fe} / \mathrm{H}]=-0.3$ to +0.2 , and were computed by finding the average difference in $M_{V}$ between the stellar values in Table 1 and the isochrone for that metallicity. The errors on the individual points are on the order of $0.01 \mathrm{mag}$. The values for the Hyades isochrone employing the Lejeune et al. (1998) color calibration are shown as filled points; the adjacent straight line is a linear least-squares fit to those points. The uncorrected Lejeune et al. (1998) and Alonso et al. $(1995,1996)$ color calibrations are displayed as open circles and triangles, respectively. The cross at $[\mathrm{Fe} / \mathrm{H}]=+0.13$ and $\Delta M_{V}=0.0$ shows where the isochrones should go if they did not require empirical corrections to the color-temperature relation (i.e., if they matched the Hyades photometry a priori.)

The center and bottom panels, respectively, plot the mean difference at fixed $V-I_{C}$ and $V-K_{s}$, computed over the same color interval in $B-V$.

The value of $\Delta M_{V}$ enters into the computation of cluster distances in several different ways. The slope of $\Delta M_{V}$ as a function of $[\mathrm{Fe} / \mathrm{H}]$ is a measure of distance errors that arise from errors in the assumed or measured metallicity of a cluster. The average slope over the range $-0.3 \leq[\mathrm{Fe} / \mathrm{H}] \leq+0.2$ is shown for the three representative color calibrations in Table 3 (the relationship is probably not precisely linear, having a somewhat steeper dependence on metallicity above $([\mathrm{Fe} / \mathrm{H}]=0.0)$. All the color calibrations have nearly the same dependence on

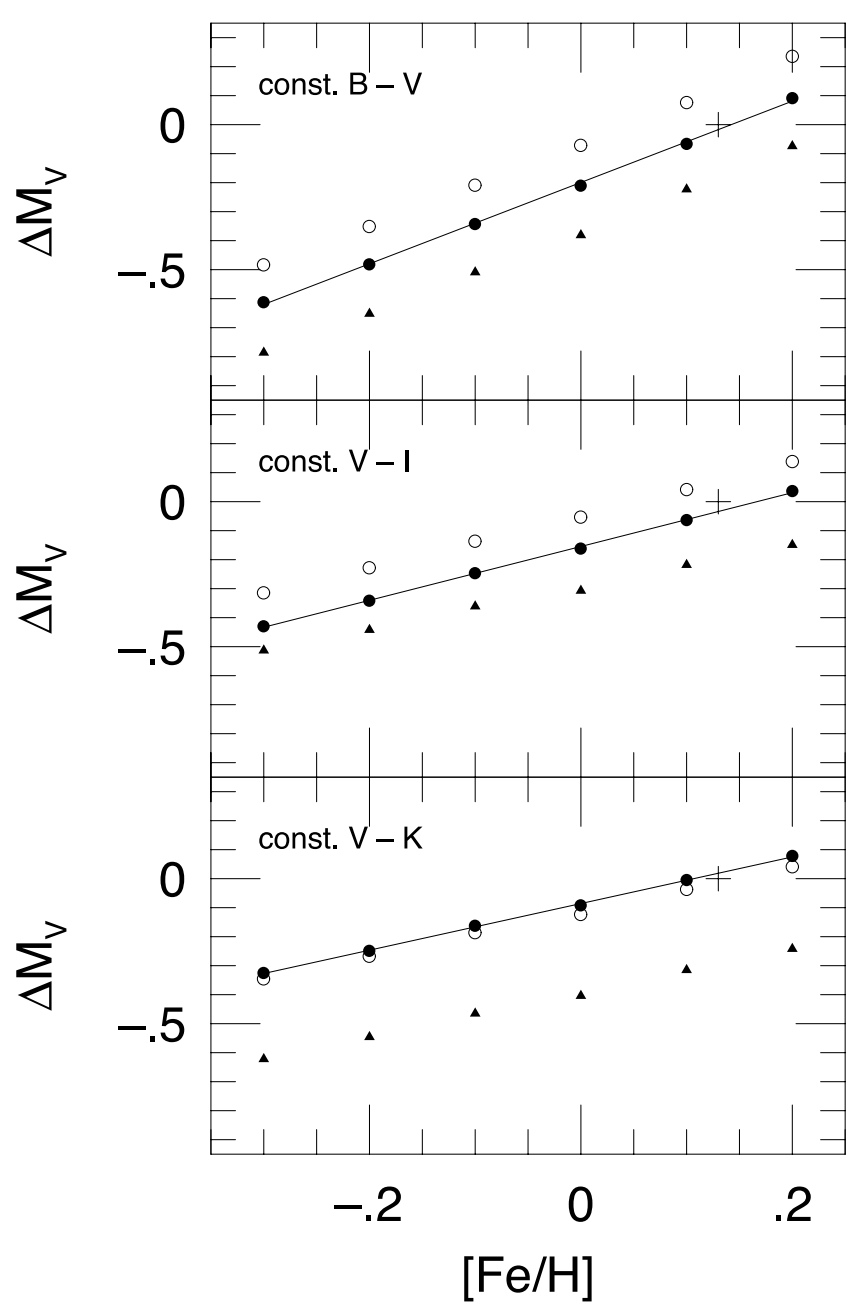

FIG. 9.-Sensitivity of the isochrone luminosity to metallicity, for the several color calibrations discussed in the text. 
TABLE 3

Metallicity Sensitivity of Isochrone

\begin{tabular}{lccccc}
\hline \hline \multicolumn{1}{c}{ Color Calibration } & $B-V$ & $V-I_{C}$ & $V-K_{s}$ & {$[\mathrm{M} / \mathrm{H}]$} & $\Delta(m-M)_{V}$ \\
\hline Lejeune et al. 1998 .............. & 1.402 & 0.927 & 0.804 & +0.142 & 0.00 \\
Uncorrected Lejeune ............ & 1.432 & 0.903 & 0.767 & -0.019 & -0.10 \\
Alonso et al. 1995, 1996..... & 1.421 & 0.727 & 0.758 & +0.036 & -0.40 \\
\hline
\end{tabular}

$M_{V}$ at fixed $B-V$, with an average slope of 1.42 mag per dex in metallicity. The metallicity dependence is less steep at fixed $V-I_{C}$ or fixed $V-K_{s}$, and we note that the Alonso et al. (1995, 1996) calibration produces a markedly shallower metallicity dependence of the MS luminosity in at constant $V-I_{C}$ than the other two calibrations.

While the various calibrations generally agree on the slope $d\left(\Delta M_{V}\right) / d[\mathrm{Fe} / \mathrm{H}]$, they have very different zero points. Since these calculations are all done for the same input physics and metallicity $([\mathrm{Fe} / \mathrm{H}]=+0.13)$, one would necessarily derive different distances to the Hyades using the various color calibrations. Forcing the distances to be the same for the various colors yields a photometric metallicity index for a cluster (e.g., Pinsonneault et al. 1998).

The last two columns of Table 3 display the value of $[\mathrm{Fe} / \mathrm{H}]$ that would be found for the various color calibrations, and the relative distance with respect to the assumed value of 3.33 for the Hyades.

\section{SUMMARY AND DISCUSSION}

In this paper we have continued our effort to improve the calibration of MS isochrones for use in determining the distances and metallicities of open clusters. We first identified a set of single Hyades stars with quality multicolor photometry, then verified that the isochrone luminosity-temperature relationship is in agreement with recent spectroscopic determinations. We then described a method for computing empirical corrections to the color-temperature relationship in the isochrone and explored the sensitivity of the MS luminosity to metallicity using several available color-temperature calibrations (see also VandenBerg and Clem 2003 for a parallel discussion of these issues). In subsequent papers, we will extend the empirical calibration to the lower MS via photometry in other clusters, determine the accuracy of photometric metallicity indicators, explore the effects of reddening, and derive ages for young systems via an analysis of the preMS/MS boundary.

The principal result of this paper was that the theoretical and spectroscopic (PSC) luminosity-temperature and luminosityradius (i.e., $L, \log g$ ) relations are in fairly good agreement (Fig. 4) except for stars with $T_{\text {eff }} \gtrsim 5800$. The spectroscopic $\log g$ values, if real, would imply large errors in the model radii that would cause much larger differences in $T_{\text {eff }}$ than seen (assuming that the mass/luminosity relationship is correct). They are also very unlikely given the similarity of the stars to the Sun, which should imply that the solar calibration produces reasonable radii for models of Hyades stars. We have verified that the dispersion in all of the abundances measured by PSC is significantly reduced when the theoretical $\log g$ values are used in place of the spectroscopic ones (a more detailed analysis will appear in a future paper). Paulson et al. note similar trends with respect to temperature scales employed by Allende Prieto \& Lambert (1999), which was based on a different set of theoretical isochrones. We suggest that there may be potential degeneracies between the spectroscopic temperature and gravity measurements. The spectroscopic $T_{\text {eff }}$ and $\log g$ values are derived from Saha/Boltzmann considerations, and the fitting procedure used by PSC may compensate for errors in the ionization balance $(\log g)$ by changing the excitation balance $\left(T_{\text {eff }}\right)$. This idea could be tested by seeing what temperature scale resulted if the gravities were taken from the theoretical isochrones rather than treating them as a free parameter. In any case, the overall agreement in between a high-quality spectroscopic data set and the isochrones in the theoretical plane provides strong support for the hypothesis that the model temperature scale is reasonable.

The main goal of this effort is to reduce the theoretical and calibration uncertainties in the theoretical models using the constraints provided by the detailed morphology of the MS in various clusters. As outlined in the $\S 1$, however, we have long been interested in the discrepancy between the Hipparcos distances to clusters (ESA 1997) as subsequently analyzed by Robichon et al. (1999), van Leeuwen (1999), and others (see Pinsonneault et al. 1998; Soderblom et al. 1998). Various attempts to explain away the discrepancies as arising from, say, strange helium abundances (Belikov et al. 1998) or stellar activity (Percival, Salaris, \& Kilkenny 2003), whether true or not (and worth exploring further), do raise an important issue: accurate calibration of isochrone physics and colors is an instrinsically difficult exercise, whereas parallaxes should be easier in principle (although perhaps not in practice).

In our view, the issue of the distance discrepancies arising from the 1997-1999 Hipparcos parallaxes is largely settled. Several papers have pointed out that small-scale errors in the parallaxes are effectively random zero-point errors over the whole sky, with an rms error of about 0.5 mas. The Hyades are less affected than other Hipparcos clusters because the Hyades subtend far larger than $1^{\circ}$, allowing the small-scale errors to average out Narayanan \& Gould (1999b). The Pleiades are strongly affected because the bright stars are quite centrally concentrated (17 Hipparcos stars per square degree at the cluster center) and so share the same zero-point error, which amounts to +1 mas at that point in the sky. Recently, Makarov (2002) has devised a method to recalculate parallaxes from the Hipparcos Intermediate Astrometric Data, free of the small angular scale-correlated errors, which are an artifact of the "great circle abscissa method" data reductions used in the ESA (1997) distances. His new Pleiades distance is in agreement with the distance from MS fitting, and also with Pleiades distances derived from kinematic parallaxes (Narayanan \& Gould 1999b) and new ground-based results (Gatewood, de Jonge, \& Han 2000).

The work reported here was supported in part by the National Science Foundation, under grants AST 97-31621 and AST 02-06008 to the Ohio State University Research Foundation. 
Allard, F., \& Hauschildt P. H. 1995, ApJ, 445, 433

Allende Prieto, C., \& Lambert, D. W. 1999, A\&A, 352, 555

Alonso, A., Arribas, S., \& Martínez-Roger, C. 1995, A\&A, 297, 197 1996, A\&AS, 117, 227

Bahcall, J. N., Pinsonneault, M. H., \& Basu, S. 2001, ApJ, 555, 990

Basu, S., Pinsonneault, M. H., \& Bahcall, J. N. 2000, ApJ, 529, 1084

Belikov, A. N., Hirte, S., Meusinger, H., Piskunov, A. E., \& Schilbach, E.

1998, A\&A, 332, 575

Bessel, M. S. 1979, PASP, 91, 589

Bessel, M. S., \& Weis, E. W. 1987, PASP, 99, 642

Boesgaard, A. M., \& Friel, E. D. 1990, ApJ, 351, 467

Carney, B. W. 1982, AJ, 87, 1527

Carney B. W., \& Aaronson M. 1979, AJ, 84, 867

Carpenter, J. M. 2001, AJ, 121, 2851

Castellani, V., Degl'Innocenti, S., \& Prada Moroni, P. G. 2001, MNRAS, 320,66

Crawford, D. L. 1975, AJ, 80, 955

de Bruijne, J. H. J., Hoogerwerf, R., \& de Zeeuw, P. T. 2001, A\&A, 367, 111

Eggen, O. J. 1948, AJ, 54, 35 1975, PASP, 87, 107 1982, ApJS, 50, 221

Elias, J. H., Frogel, J. A., Matthews, K., \& Neugebauer, G. 1982, AJ, 87, 1029

European Space Agency, 1997, The Hipparcos and Tycho Catalogues (ESA SP-1200; Paris: ESA)

Flower, P. J. 1996, ApJ, 469, 355

Frogel, J. A., Persson, S. E., Aaronson, M., \& Matthews, K. 1978, ApJ, 220,75

Gatewood, G., de Jonge, J. K., \& Han, I. 2000, ApJ, 533, 938

Gratton, R. G., Carretta, E., \& Castelli, F. 1996, A\&A, 314, 191

Grevesse, N., \& Noels, A. 1993, in Origin and Evolution of the Elements, ed. M. Prantzos, E. Vangioni-Flam, \& M. Cassé (Cambridge: Cambridge Univ. Press), 15

Grocholski, A. J., \& Sarajedini, A. 2003, MNRAS, 345, 1015

Johnson, H. L., \& Knuckles, C. F. 1955, ApJ, 122, 209

Johnson, H. L., MacArthur J. W., \& Mitchell R. I. 1968, ApJ, 152, 465

Johnson, H. L., Mitchell R. I., Iriarte B., \& Wisniewski W. Z. 1966, Commun. Lunar. Planet. Lab. 4, 99

Lejeune, T., Cuisinier, F., \& Buser, R. 1998, A\&AS, 130, 65

Lejeune, T., \& Schaerer, D. 2001, A\&A, 366, 538

Makarov, V. V. 2002, AJ, 124, 3299

2003, AJ, 126, 2408

Mendoza, E. E. 1967, Tonantzintla Tacubaya, 4, 149

Mermilliod, J.-C., Turon, C., Robichon, N, Arenou, F., \& Lebreton, Y. 1997, in Hipparcos Venice '97, ed. B. Battrick \& M. A. C. Perryman (ESA SP-402; Paris: ESA), 643
REFERENCES

Narayanan, V. K., \& Gould, A. 1999a, ApJ, 515, 256 1999b, ApJ, 523, 328

Paulson, D. B., Sneden, C., \& Cochran, W. D. 2003, AJ, 125, 3185 (PSC)

Percival, S. M., Salaris, M., \& Kilkenny, D. 2003, A\&A, 400, 541

Perryman, M. A. C., et al. 1998, A\&A, 331, 81

Persson, S. E., Murphy, D. C., Krzeminski, W., Roth, M., \& Rieke, M. J. 1998, AJ, 116, 2475

Pinsonneault, M. H., Stauffer, J., Soderblom, D. R., King, J. R., \& Hanson, R. B. 1998, ApJ, 504, 170

Pinsonneault, M. H., Terndrup, D. M., Hanson, R. B., \& Stauffer, J. R. 2003, ApJ, 598, 588 (Paper I)

Robichon, N., Arenou, F., Mermilliod, J.-C., \& Turon, C. 1999, A\&A, 345,471

Robichon, N., Lebreton, Y., Turon, C., \& Mermilliod, J.-C. 2000, in ASP Conf. Ser. 198, Stellar Clusters and Associations: Convection, Rotation, and Dynamos, ed. R. Pallavicini, G. Micela, \& S. Sciortino (San Francisco: ASP), 141

Saumon, D., Chabrier, G., \& Van Horn, H. M. 1995, ApJS, 99, 713

Soderblom, D. R., King, J. R., Hanson, R. B., Jones, B. F., Fischer, D., Stauffer, J. R., \& Pinsonneault, M. H. 1998, ApJ, 504, 192

Stauffer, J. R., Jones, B. F., Backman, D., Hartmann, L. W., Barrado y Nevascués, D., Pinsonneault, M. H., Terndrup, D. M., \& Muench, A. 2003, AJ, 126, 833

Stello, D., \& Nissen, P. E. 2001, A\&A, 374, 105

Taylor, B. J. 1980, AJ, 85, 242

Taylor, B. J., \& Joner, M. D. 1985, AJ, 90, 479

Terndrup, D. M., Pinsonneault, M. H., Jeffries, R. D., Ford, A., Stauffer, J. R., \& Sills, A. 2002, ApJ, 576, 950

Terndrup, D. M., Stauffer, J. R., Pinsonneault, M. H., Sills, A., Yuan, Y., Jones, B. F., Fischer, D., \& Krishnamurthi, A. 2000, AJ, 119, 1303

Upgren, A. R., \& Weis, E. W. 1977, AJ, 82, 978

Upgren, A. R., Weis, E. W., \& Hanson, R. B. 1985, AJ, 90, 2039

VandenBerg, D. A., \& Clem, J. L. 2003, AJ, 126, 778

VandenBerg, D. A., Swenson, F. J., Rogers, F. J., Iglesias, C. A., \& Alexander, D. R. 2000, ApJ, 532, 430

van Leeuwen, F. 1999, A\&A, 341, L71

van Leeuwen, F., Alphenaar, P., \& Meys, J. J. M. 1987, A\&AS, 67, 483

van Leeuwen, F., \& Evans, D. W. 1998, A\&AS, 130, 157

Weiss, A., \& Salaris, M. 1999, A\&A, 346, 897

Weis, E. W., Deluca, E. E., \& Upgren, A. R. 1979, PASP, 91, 766

Weis, E. W., \& Hanson, R. B. 1988, AJ, 96, 148

Weis, E. W., \& Upgren, A. R. 1982, PASP, 94, 475

Yi, S. K., Kim, Y.-C., \& Demarque, P. 2003, ApJS, 144, 259 